\title{
Lack of Flvcr2 impairs brain angiogenesis without affecting the blood-brain barrier
}

\author{
Nicolas Santander, ${ }^{1}$ Carlos O. Lizama, ${ }^{2}$ Eman Meky, ${ }^{1}$ Gabriel L. McKinsey, ${ }^{1}$ Bongnam Jung, ${ }^{3}$ Dean Sheppard, ${ }^{4}$ Christer Betsholtz, ${ }^{3,5}$ \\ and Thomas D. Arnold' \\ 'Department of Pediatrics, and 2 Cardiovascular Research Institute, UCSF, San Francisco, California, USA. Integrated Cardiometabolic Center, Department of Medicine, Huddinge, Karolinska Institutet, Solna, \\ Sweden. ㅁepartment of Cell Biology, UCSF, San Francisco, California, USA. 5Department of Immunology, Cenetics and Pathology, Uppsala University, Uppsala, Sweden.
}

\begin{abstract}
Fowler syndrome is a rare autosomal recessive brain vascular disorder caused by mutation in FLVCR2 in humans. The disease occurs during a critical period of brain vascular development, is characterized by glomeruloid vasculopathy and hydrocephalus, and is almost invariably prenatally fatal. Here, we sought to gain insights into the process of brain vascularization and the pathogenesis of Fowler syndrome by inactivating Flvcr2 in mice. We showed that Flvcr2 was necessary for angiogenic sprouting in the brain, but surprisingly dispensable for maintaining the blood-brain barrier. Endothelial cells lacking Flvcr2 had altered expression of angiogenic factors, failed to adopt tip cell properties, and displayed reduced sprouting, leading to vascular malformations similar to those seen in humans with Fowler syndrome. Brain hypovascularization was associated with hypoxia and tissue infarction, ultimately causing hydrocephalus and death of mutant animals. Strikingly, despite severe vascular anomalies and brain tissue infarction, the blood-brain barrier was maintained in Flvcr2 mutant mice. Our Fowler syndrome model therefore defined the pathobiology of this disease and provided new insights into brain angiogenesis by showing uncoupling of vessel morphogenesis and blood-brain barrier formation.
\end{abstract}

\section{Introduction}

Developmental vascularization, the process by which blood vessels grow into and differentiate within a tissue, is remarkably organ specific. Cerebral angiogenesis is the foremost example. Here, blood vessels sprout from a primitive perineural plexus into the brain parenchyma until they reach the lateral ventricles, then grow laterally to form a secondary plexus spanning the ventricular zone (1). As vessels grow into the brain they coordinately acquire barrier properties (i.e., the blood-brain barrier [BBB]), which are a unique and defining feature of the CNS vasculature. Indeed, mRNA profiling studies reveal significant differences between endothelial cells in the brain and in other organs $(2,3)$. Many of these "BBB-specific" genes encode transporters or cellular junction proteins, and mouse mutants lacking some of these genes (e.g., $M f s d 2 a, L s r, C l d n 5$; refs. 4-6) have isolated BBB dysfunction. On the other hand, disruption of $\alpha \mathrm{V} \beta 8 / \mathrm{TGF}-\beta$ or Wnt $/ \beta$-catenin signaling during development produces CNS-specific angiogenic abnormalities and concomitant BBB dysfunction $(7,8)$, suggesting that downstream programs controlling cerebral angiogenesis and barrier genesis are overlapping. The genes regulating cerebral angiogenesis independently of the BBB are entirely unknown; to the best of our knowledge, there are as yet no mouse models that display abnormal vascular growth and, at the same time, an intact $\mathrm{BBB}$. The discovery of such a model would be immensely useful

Conflict of interest: The authors have declared that no conflict of interest exists. Copyright: () 2020, American Society for Clinical Investigation.

Submitted: January 21, 2020; Accepted: April 22, 2020; Published: June 22, 2020.

Reference information: J Clin Invest. 2020;130(8):4055-4068.

https://doi.org/10.1172/JCl136578. in contributing to our understanding of how vascular growth and differentiation are specifically regulated in the CNS and could help determine the downstream consequences of abnormal CNS angiogenesis versus BBB dysfunction.

In humans, mutations in the poorly studied gene FLVCR2 (also known as MFSD7C, SLC49A2, and CCT) underlie the development of proliferative vasculopathy with hydranencephalyhydrocephaly syndrome (PVHH) (OMIM 225790), also known as Fowler syndrome (9). This gene is part of the major facilitator superfamily and has been proposed as a putative heme importer $(10,11)$. Its expression is restricted to brain endothelial cells (3), suggesting a role in the barrier properties of these cells.

$\mathrm{PVHH}$ is an autosomal recessive prenatal lethal disorder characterized by severe hydrocephalus and thin cerebral cortex, glomeruloid vascular structures in the brain, and diffuse ischemic lesions in the brain stem and embryonic basal ganglia or ganglionic eminence (GE) (12). To date, there are 16 different reported FLVCR2 mutations in humans: 1 large deletion, 2 nonsense mutations, 1 splice-site mutation, 1 insertion/deletion change, and 11 missense variations $(9,13,14)$. These are most likely loss-of-function mutations, since heterozygotes are not normally affected and, because most affected individuals are compound heterozygotes, having 2 different recessive alleles at the FLVCR2 locus. While the exact consequences of these mutations have not yet been tested in existing functional assays (11), multiple-sequence alignment and homology modeling support local conformational changes (usually affecting transmembrane domains with predicted dysfunction of the channel) or reduced expression (null mutations) (13). Furthermore, in the genome aggregation database (gnomAD, gnomad.broadinstitute.org), no homozygous loss-of-function 
A
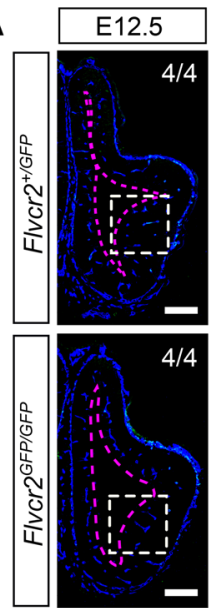
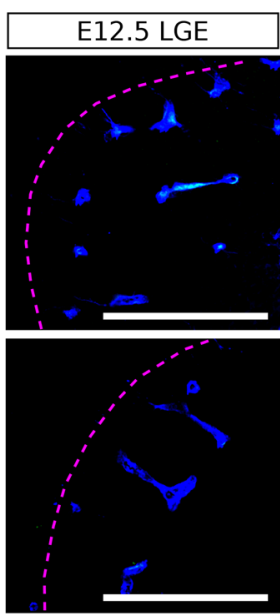
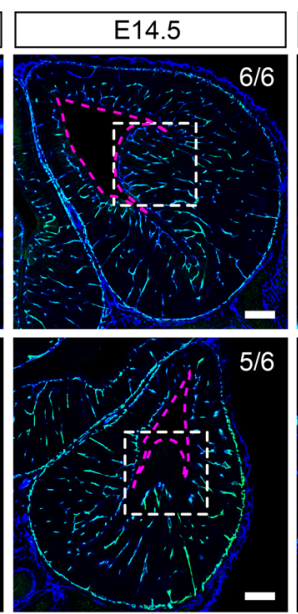

E14.5 LGE
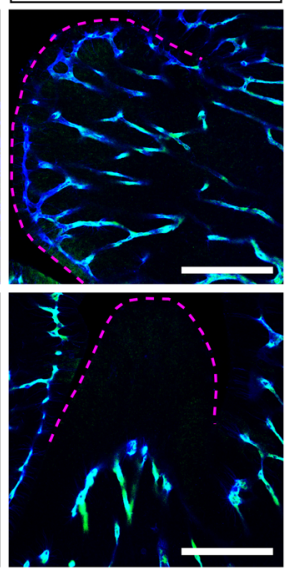
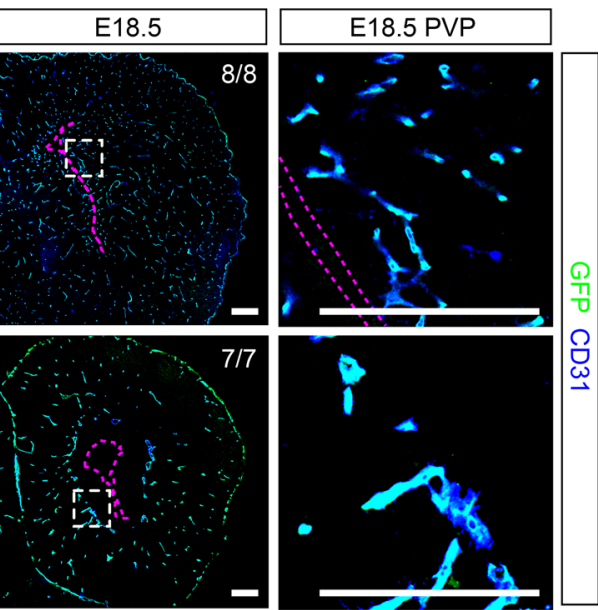

B

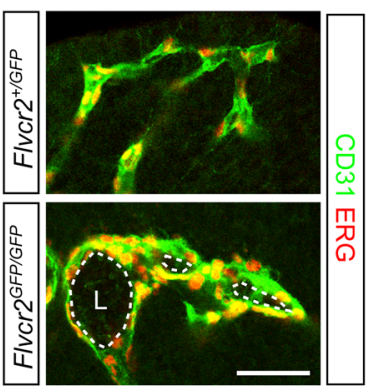

c

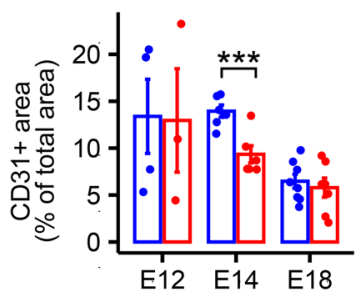

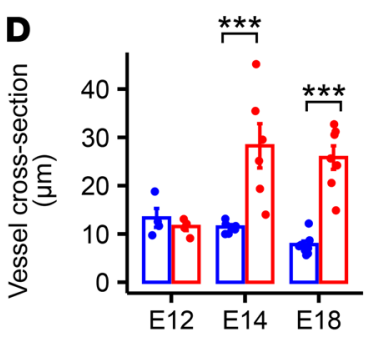
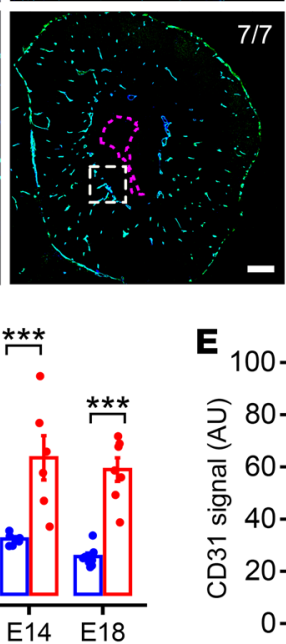

口FIVCr2 ${ }^{+/ G F P}$ $\square$ FIVCr2 $2^{\text {GFP/GFP }}$
F

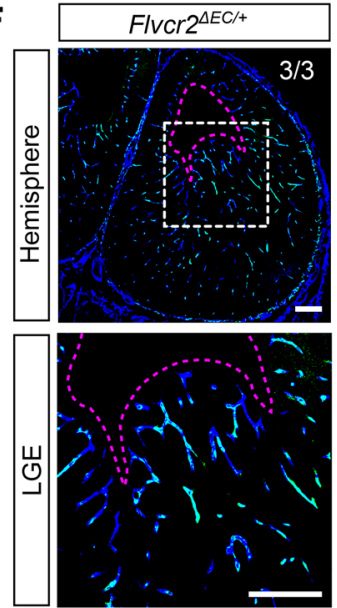

Flvcr $2^{\triangle E C / \Delta E C}$
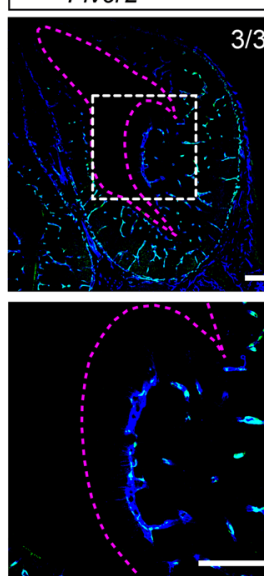

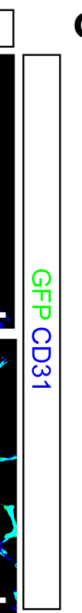

$\mathbf{G}$

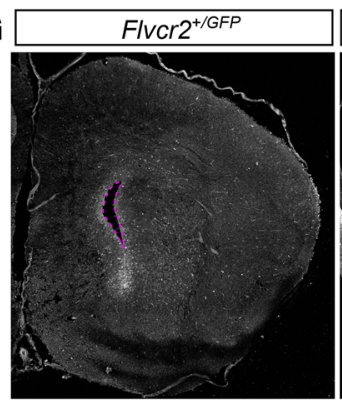

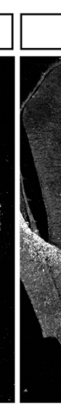

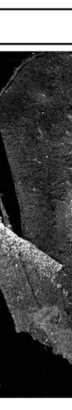

Flver

P/GFP

Figure 1. Flvcr2 inactivation in mice models human PVHH. (A) Coronal sections through the lateral ventricles at the indicated embryonic stages were stained to visualize GFP and the endothelial cell marker CD31. Dotted violet lines delineate the ventricles. Boxed areas show magnified areas of the LGE. Numbers indicate the number of embryos with the represented phenotype. Scale bars: $200 \mu \mathrm{m}$. (B) Vessels in embryonic brains were stained with CD31 and ERG antibodies. A representative dilated vessel (from 5 E14.5 embryos with the phenotype) in the Flvcr2 ${ }^{\mathrm{CF} / \mathrm{CF} F} \mathrm{CE}$ is shown. L, vessel lumen. Scale bar: $50 \mu \mathrm{m}$. (C) Fraction of brain area covered by CD31+ cells. ${ }^{* * *} P<0.001, t$ test. E12.5, $n=4$; E14.5, $n=6$; E18.5, $n=7-8$. (D) Thickness of periventricular vessels. ${ }^{* *} P<0.001, t$ test. E12.5, $n=4$; E14.5, $n=6$; E18.5, $n=7-8$. (E) Fluorescence intensity profile of CD31 in the LGE of Flvcr2 ${ }^{+/ C F P}$ and Flvcr2 ${ }^{C F P / C F P}$ embryos at E14.5 expressed as mean (solid line) + SEM (shaded area). $n=6$. (F) Coronal sections of E14.5 brains were obtained from embryos with constitutive (Flvcr ${ }^{4 E C}$ ) deletion of Flvcr2 in endothelial cells and stained for GFP and CD31. Scale bars: $200 \mu \mathrm{m}$. (C) Coronal sections of E18.5 brains were stained for SOX9 (a marker of apical neuro/glia progenitors at this stage) to reveal the ventricle wall. Scale bar: $500 \mu \mathrm{m}$. (H) Ventricle area was compared in mutants and controls. ${ }^{*} P<0.05, t$ test. Flver2 ${ }^{+/ G F P}, n=8$; FlvCr2 ${ }^{\text {CFP/GFP }}, n=7$.

mutation is reported in the FLVCR2 locus in over 125,000 exomes and 15,000 genomes from adults, again suggesting that loss-offunction mutations (and not potentially activating mutations) are responsible for PVHH. Due to the rarity of the syndrome and the lack of a good animal model, the step-wise progression of vascu- lar abnormalities, hydrocephalus, and embryonic lethality and the contribution of BBB dysfunction to this pathology are not known.

Here, we have generated a mouse model of PVHH by inactivating mouse Flvcr2 in an effort to better understand brain vascular and BBB codevelopment and to deconstruct the molecular 


\section{Table 1. Number of WT, Flvcr2 ${ }^{+/ G F P}$, and Flvcr2 ${ }^{\text {GFP/GFP embryos }}$ and pups recovered at different developmental stages}

$\begin{array}{lccc}\text { Age } & \text { WT } & \text { Flvcr2 }^{+/ / \text {FP }} & \text { Flvcr2 }^{\text {CFP//FP }} \\ \text { E12.5 } & 3 & 5 & 4 \\ \text { E14.5 } & 7 & 16 & 10 \\ \text { E18.5 } & 7 & 16 & 9 \\ \text { P28 } & 51 & 95 & 0^{\text {A }}\end{array}$

${ }^{\mathrm{A} P}<0.0001, \chi^{2}$ test.

pathophysiology of this disease. We show that Flver 2 is specifically expressed in CNS endothelial cells throughout development and into adulthood and that inactivation of Flvcr 2 in these cells severely impairs brain vascular growth. Surprisingly, knockout mice have an intact $\mathrm{BBB}$, suggesting that the downstream phenotypes observed in mice and humans with mutant FLVCR2 (hydrocephalus and embryonic lethality) are a consequence of abnormal brain angiogenesis and not BBB dysfunction. These observations disentangle the codevelopment of the $\mathrm{BBB}$ and brain blood vessels and describe distinct downstream consequences of impaired vascular growth in the brain.

\section{Results}

Flvcr 2 is selectively expressed in CNS endothelium and pericytes. $\mathrm{PVHH}$ is a rare autosomal recessive and lethal genetic disorder caused by mutations in the FLVRC2 gene in humans (9). However, the natural history, pathological progression, and mechanisms driving the phenotype in this disease are unknown. In previous RNA-Seq studies, we and others found Flvcr2 to be highly expressed at the BBB in mice relative to other organ vasculature (Supplemental Figure 1 and refs. 3, 15; supplemental material available online with this article; https://doi.org/10.1172/JCI136578DS1). To confirm these results, we generated tools to study the expression pattern of the gene and its function in vivo. To assess the expression of the Flver 2 locus, we generated an Flvcr2 conditional knockin/knockout EGFP reporter mouse (hereafter termed Flvcr $2^{\text {GFP }}$; Supplemental Figure 1A). In this mouse line, the Flvcr 2 locus was mutated by targeted recombination to insert loxP sites flanking the second exon of the Flvcr2 gene and an inverted GFP cassette, which was also flanked by mutated loxP sites. Upon Cre-mediated recombination, the GFP cassette was irreversibly flipped in-frame, while the second exon was removed, leading to the inactivation of the gene and the expression of GFP under the control of the endogenous locus. To generate a knockout (null) allele, we crossed the floxed line with mice carrying a Cre transgene expressed under the control of the $M e f 2 c$ promoter, which recombined the floxed allele in the germline.

We found that Flvcr 2 mRNA (as assessed by the GFP reporter) was most highly expressed in the brain compared with other major organs in adult mice (Supplemental Figure 2). We also detected GFP reporter expression in presumed alveolar macrophages in the lung and sparse signal in intestinal epithelial cells and hepatocytes (Supplemental Figure 2). Consistent with published bulk and single cell expression data sets (Supplemental Figure 3A and ref. 16), we observed GFP expression in all vascular segments (i.e., arter- ies, veins, and capillaries) in a pattern consistent with endothelial cells in adult animals (Supplemental Figure 3B) as well as in embryos (Supplemental Figure 3C). Since Flvcr 2 expression is also detected in pericytes in a single-cell transcriptomic data set, albeit at lower level and/or in a smaller subset of the cells (16), it is possible that some of the GFP signal emanates from pericytes; however, this would be hard to unequivocally establish, given the stronger or more prevalent expression in the neighboring endothelial cells.

$\mathrm{PVHH}$ is a distinctly developmental disorder, affecting mid- to late- gestation fetuses. We used our Flvcr ${ }^{G F P}$ reporter line to track expression during embryonic development. We first detected GFP in a subset of brain endothelial cells around E12.5 (Figure 1A). Subsequently, both the number and proportion of GFP-expressing brain endothelial cells increased until E18.5, at which time most CNS endothelium was $\mathrm{GFP}^{+}$(Figure 1B). We also detected GFP signal in the syncytiotrophoblasts of the placenta and yolk sac visceral endoderm (Supplemental Figure 3C), but not in any other tissues in the embryo proper. Finally, flow cytometry analysis of cells from whole E14.5 brains (Supplemental Figure 4A) confirmed that the vast majority of the $\mathrm{GFP}^{+}$cells were also $\mathrm{CD} 31^{+}$; however, about $10 \%$ of these were GFP negative, potentially corresponding to Flvcr2-positive pericytes. Embryonic blood cells from Flvcr2 ${ }^{G F P}$ mice did not express GFP (not shown).

Reduced brain vascularization in Flvcr 2 mutant mice. To investigate the function of Flvcr2 in mice, we generated mouse knockouts by intercrossing heterozygous $F l v c r 2^{+/ G F P}$ mice. Flvcr2 $2^{+/ G F P}$ mice were born at Mendelian frequencies and were healthy and fertile; however, we failed to recover nullizygous Flvcr $2^{\text {GFP/GFP }}$ mice at weaning age, demonstrating that Flvcr2 gene inactivation causes embryonic or early postnatal lethality (Table 1 ). Newborn Flvcr $2^{\text {GFP/GFP }}$ pups could only survive for a few hours after birth, with signs of severe hypoxia (cyanosis, reduced movement). We did not observe any gross malformation in mutant embryos.

We next focused on brain vascular phenotypes in mutant embryos. Flow cytometry analysis of brain endothelial cells from embryos at E15.5 confirmed increased GFP signal in Flvcr $2^{\text {GFP/GFP }}$ samples compared with Flvcr2 ${ }^{+/ G F P}$ endothelium (Supplemental Figure 4D). Coronal sections through $\mathrm{FlvCr}^{+/ G F P}$ and Flvcr2 ${ }^{\text {GFP/GFP }}$ brains revealed striking vascular malformations in the median and lateral GEs (LGEs) (Figure 1, A and B). Time-course analyses indicated that periventricular vessels in Flvcr $2^{\text {GFP/GFP }}$ embryos were apparently normal at E12.5 (an early stage of vascular growth in the brain), but became progressively hypoplastic from E14.5 until E18.5. Vascular malformations led to a reduction in the area of the brain periventricular parenchyma covered by vessels (Figure 1C) and dilation of vascular tubes with "clumping" of $\mathrm{ERG}^{+}$endothelial nuclei within vessel walls (Figure 1, B and D). Furthermore, these vessels appeared to stall in their ingression toward the ventricular zone, forming a vascular plexus approximately 300 $\mu \mathrm{m}$ from the ventricles compared with the periventricular plexus (PVP), which normally forms immediately adjacent to the ventricular surface (Figure 1E). The localization of vascular anomalies to the periventricular vascular plexus suggests that vessels in this region are particularly sensitive to Flvcr2 gene disruption. The vascular phenotype and embryonic lethality were recapitulated in mice with endothelial cell-specific deletion of Flvcr2, generated by crossing floxed mice with animals carrying a Cre recombinase 


\begin{tabular}{|c|c|c|c|c|}
\hline Age & $\mathrm{fl} /+$ & fl/+;Cre & $f l / f l$ & fl/fl;Cre \\
\hline E12.5 & 9 & 10 & 11 & 10 \\
\hline E14.5 & 5 & 7 & 8 & 5 \\
\hline P28 & 32 & 30 & 44 & $0^{A}$ \\
\hline
\end{tabular}

${ }^{A} P<0.0001, \chi^{2}$ test.

transgene under the control of the Tek promoter $\left(\mathrm{Flvcr}^{\mathrm{fl} / \mathrm{fl}} ; \mathrm{Tek}\right.$ $\mathrm{Cre}$, referred to as Flvcr $2^{\triangle E C}$ ) (Figure $1 \mathrm{~F}$ and Table 2), confirming that Flvcr2 largely functions in brain endothelial cells, as would be predicted based on the Flvcr2 expression pattern. Of note, we observed enlarged ventricles in Flvcr $2^{\mathrm{GFP} / \mathrm{GFP}}$ embryos at E18.5 (Figure 1, G and H), reminiscent of the hydrocephalus phenotype observed in humans with PVHH.

Reduced vascular growth in brains lacking Flvcr2 leads to hypoxia and cell death. We next sought to evaluate the pathological consequences of impaired vascularization in the GE. We hypothesized that impaired angiogenesis and reduced cerebral vascularization in $F l v c r 2^{G F P / G F P}$ mutants might lead to hypoperfusion of the brain and subsequent hypoxia and tissue infarction. Consistent with this model, we observed a strong signal of pimonidazole (hypoxyprobe) adducts in GE regions devoid of blood vessels in Flvcr2 ${ }^{\text {GFP/GFP }}$ embryos at E14.5 and E18.5 (Figure $2, \mathrm{~A}$ and $\mathrm{B}$ ), indicating the presence of low oxygen tension in these avascular tissues. Hypoxia normally induces angiogenesis largely through the activation of HIF-1 $\alpha$ translocation to the nucleus, promoting its activity as a transcription factor and leading to the expression of VEGF (17). We observed increased HIF-1 $\alpha$ nuclear staining specifically in the progenitor population located in the avascular GE of Flvcr $2^{\text {GFP/GFP }}$ embryos (Figure 2C), concomitant with a striking increase in VEGF-A immunoreactivity in the same area, compared with the well-vascularized GE of $\mathrm{Flvcr2}^{+/ \text {GFP }}$ control embryos (Figure 2D). Unabated hypoxia from reduced vascularization causes metabolic collapse, tissue infarction, and cell death. Using active caspase-3 immunostaining, we observed massive cell death in the ventral parts of the brain of Flvcr $2^{\text {GFP/GFP }}$ embryos at E18.5 (Figure 2E) concentrated in areas lacking blood vessels. Interestingly, there were relatively few apoptotic endothelial cells in the brain, and there was little apoptosis observed at early time points (E14.5), even in the presence of vascular malformation and tissue hypoxia. These observations suggest that endothelial cell apoptosis is an unlikely mechanism underlying vascular malformation and downstream sequelae in $F l v c r 2^{\text {GFP/GFP }}$ mice. Together, these data support our model showing that impaired vascular growth in brains of $F l v c r 2^{\text {GFP/GFP }}$ embryos leads to hypoxia and tissue infarction, providing a possible explanation for the ultimate death of the mutant animals.

After tissue ischemia/infarction in the brain and other organs (e.g., heart), monocytes/macrophages rapidly accumulate within the damaged tissue, leading to the production of inflammatory mediators $(18,19)$. These cells can directly affect vascular morphogenesis and neurovascular integrity $(20,21)$. Indeed, we observed a temporal trend in Flvcr2 ${ }^{\text {GFP/GFP }}$ embryos to accumulate myeloid cells in the brain, which was not seen in control embryos (Supplemental Figure 5, A and B). To assess the relevance of increased myeloid cell population in the embryo brain, we depleted them by pharmacological inhibition of CSF1R with PLX5622 in 1 litter (22). We did not detect any IBA1 $1^{+}$PU. $1^{+}$cells in the embryos from this litter at E18.5 (Supplemental Figure 5C), indicating successful depletion of the myeloid lineage. However, the phenotypic consequences of Flvcr2 inactivation were present; we observed severe vascular malformations and massive cell death in the brain of Flvcr ${ }^{\text {GFP/GPP }}$ embryos (Supplemental Figure 5D). This result suggests that increased infiltration of macrophages does not drive the alterations found in Flvcr $2^{\text {GFP/GFP }}$ embryos.

Flvcr2 inactivation impairs angiogenic sprouting. Within the periventricular vascular plexus, vascular growth is predominantly mediated by sprouting angiogenesis $(23,24)$, wherein endothelial cells of the vascular front (tip cells) extend filopodia into avascular regions toward the cerebral ventricles and initiate anastomosis with neighboring tip cells. This process is driven by tissue-derived VEGF gradients, which signal through endothelial VEGFR2 (encoded by the $K d r$ gene), and further regulated by various other pathways that can influence VEGF signaling, such as DLL4NOTCH, NRP1, and WNT, among others $(25,26)$.

To understand the molecular alterations in endothelial cells associated with Flvcr2 inactivation, we sought to define their transcriptional landscape in Flvcr2 $2^{\text {GFP/GFP }}$ embryos. Using FACS, we isolated $\mathrm{GFP}^{+} \mathrm{CD} 31^{+}$cells from embryonic brains at E15.5 and defined their transcriptional profile by RNA-Seq. Within this transcriptomic data, we detected differential expression in 28 genes involved in sprouting angiogenesis in $F l v c r 2^{G F P / G F P}$ mutants compared with Flvcr ${ }^{+/ G F P}$ controls ( $25 \%$ of genes associated with sprouting angiogenesis in the gene ontology [GO] database; Figure 3A and Table 3), suggesting a regulatory role for $\mathrm{Flvcr} 2$ in the brain angiogenic program. Interestingly, by reanalyzing single-cell transcriptomic data from chronically hypoxic brain (27), we found some of these genes were upregulated in a hypoxia-specific population of endothelial cells (Figure 3B), including Dll4, Esm1, and Flt4 (which codes for VEGFR3). This suggests that hypoxia may drive the transcriptional response observed in Flvcr2 $2^{\text {GPP/GFP }}$ endothelial cells. Next, we evaluated the protein levels of these genes by Western blot and/or immunofluorescence (Figure 3, C-E). For the total protein analyses by Western blot, we isolated vascular fragments from E15.5 brains to enrich for endothelial cells and reduce possible signals from neural progenitors. At the whole-brain vasculature level, we observed an increase in the protein levels of DLL4 and VEGFR3 (Figure 3B). We next assessed whether these proteins were increased specifically in the LGE using immunofluorescence. As expected, DLL4 and ESM1 were localized in the more tip-like cells of the LGE PVP in control $\mathrm{Flvcr}^{+/ G F P}$ embryos (Figure 3C), in sharp contrast with mutant embryos, in which these proteins localized to endothelial cells of the malformed vessels, deeper into the LGE (Figure 3C). In addition, the fluorescence intensity of VEGFR3 and ESM1 were increased in the endothelial cells of the LGE from $\mathrm{Flvcr}^{\mathrm{GFP} / \mathrm{GPP}}$ embryos compared with those in $\mathrm{Flvcr}^{+/ G F P}$ controls (Figure 3D).

Mice with inactivation of WNT $\beta$-catenin signaling or TGF- $\beta$ signaling have brain vascular phenotypes and ventriculomegaly similar to that observed in Flvcr2 mutants $(7,8,28)$. To assess whether these 

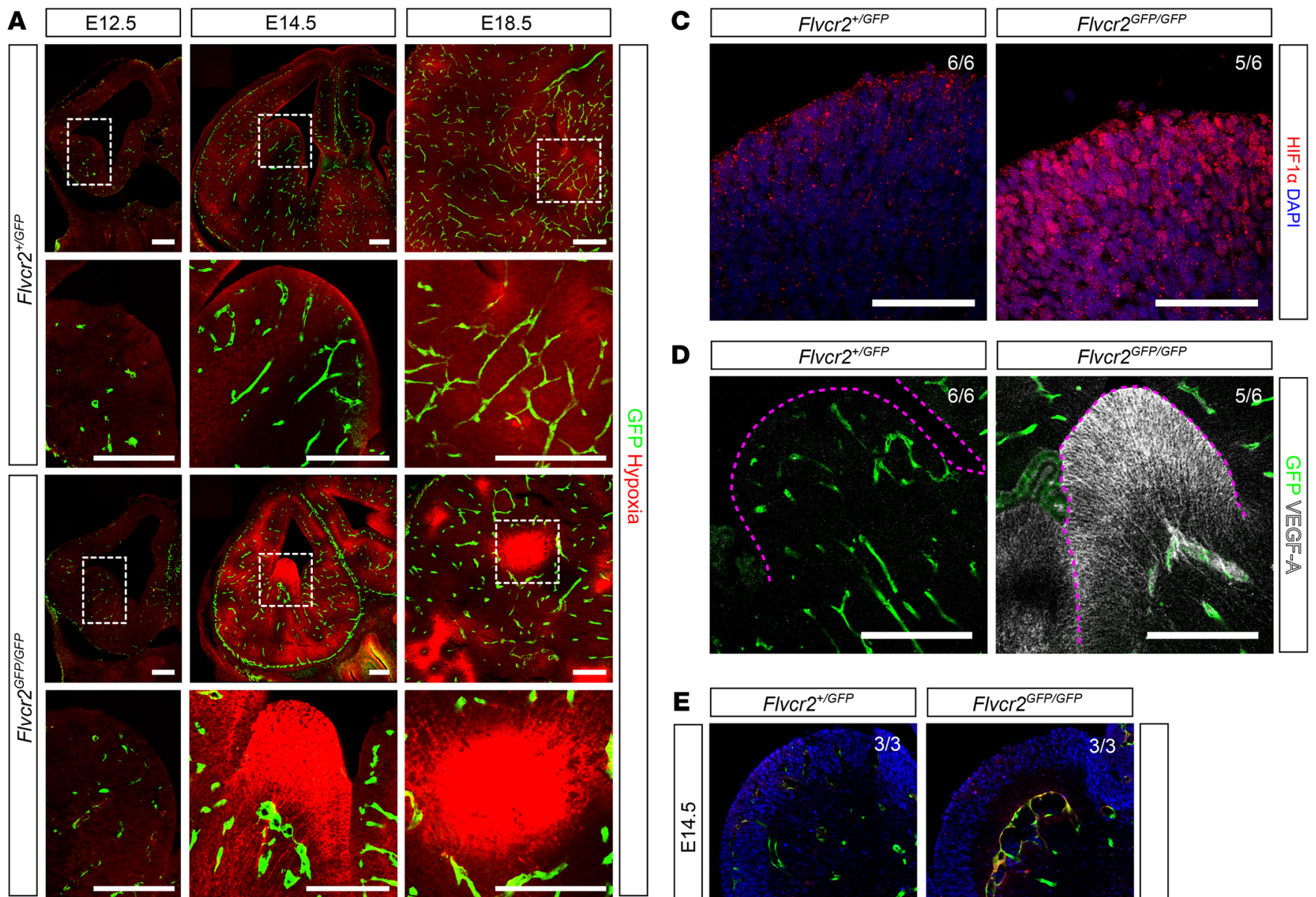

D
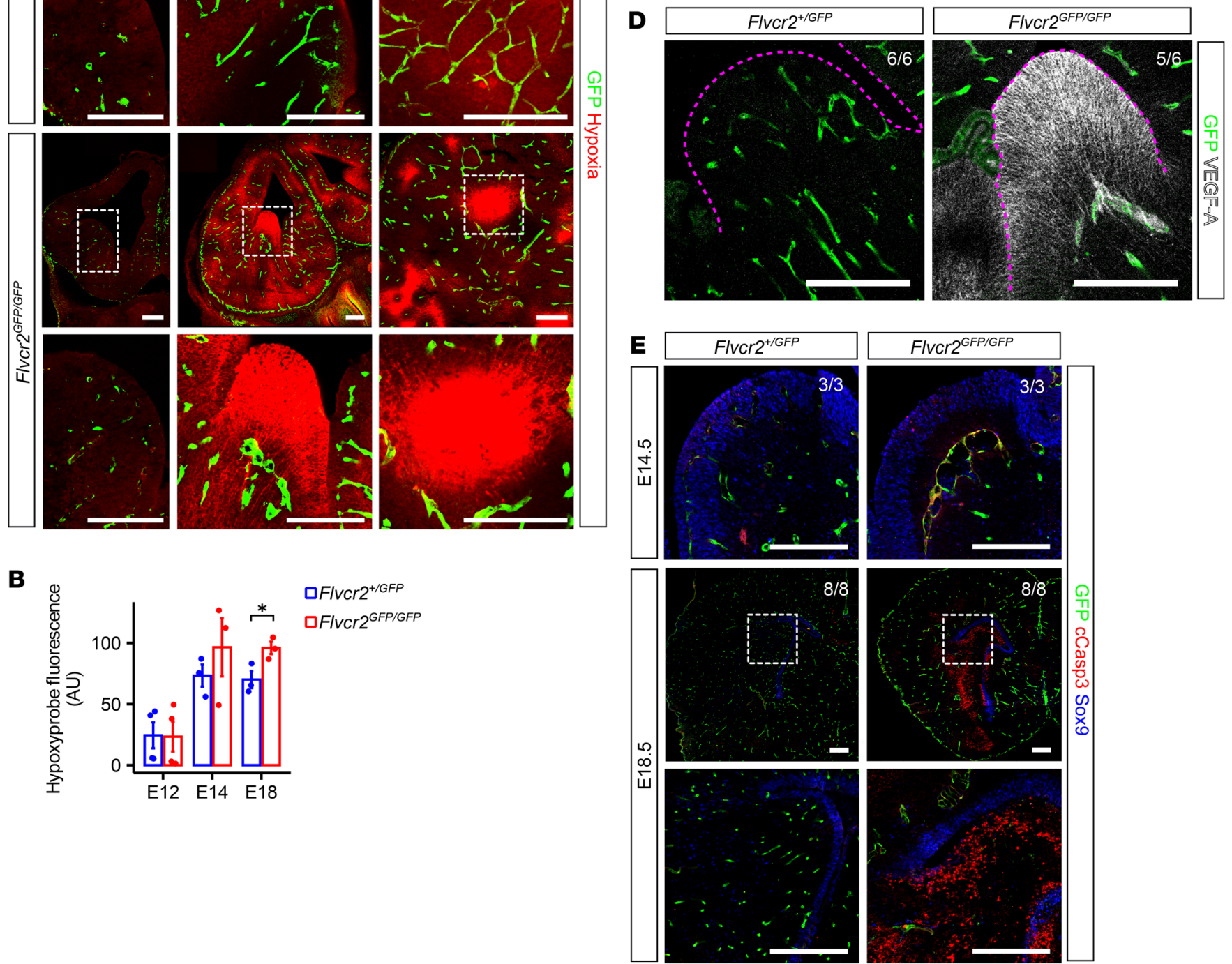

Figure 2. Brain pathology associated with Flvcr2 deletion. (A) Pregnant dams were injected with pimonidazole i.p. 3 hours before embryo collection. Pimonidazole adducts in hypoxic regions were detected in sections with a specific antibody. Scale bars: $200 \mu \mathrm{m}$. (B) Fluorescence intensity corresponding to pimonidazole adducts was quantified in the whole hemisphere. ${ }^{*} P<0.05, t$ test. E12.5, $n=4$; E14.5, $n=3 ;$ E18.5, $n=3$. (C) E14.5 sections containing the LGE were stained for HIF1 $\alpha$ and DAPI. Representative images from 6 Flvcr2 ${ }^{+/ G F P}$ and 5 Flvcr2 ${ }^{\text {CFP/CFP }}$ embryos. Scale bars: $50 \mu \mathrm{m}$. (D) LGE sections were stained to reveal the presence of VEGF-A with a specific antibody. Violet dashed lines demarcate the GE. Representative images from 6 Flvcr2 ${ }^{+/ G F P}$ and 5 Flvcr $2^{\text {CFP/GFP }}$ embryos. Scale bars: $200 \mu \mathrm{m}$. (E) Coronal sections from E14.5 and E18.5 were stained for activated caspase-3 to mark dying cells. SOX9 was used to delineate the ventricular zone. Boxed regions indicate the area magnified in the lower images. Representative images from 6 Flvcr2 ${ }^{+/ C F P}$ and 5 Flvcr ${ }^{\text {CFP/CFP }}$ embryos at E14.5 and 8 FlvCr2 $2^{+/ G F P}$ and 7 Flvcr2 ${ }^{\text {CFP/GFP }}$ embryos at E18.5. Scale bars: $200 \mu \mathrm{m}$.

pathways were affected in Flvcr2 mutants, we performed Western blot (from brain vascular fragments) and immunohistochemistry probing for downstream targets in these pathways. These experiments indicated that phosphorylated SMAD3 (a canonical TGF- $\beta$-signaling target) was unchanged in endothelial cells from Flvcr2 mutants (Supplemental Figure 6, A and B). Similarly, using Western blot for AXIN2 (a direct target of WNT/ $\beta$-catenin signaling; ref. 29) and a WNT/ $\beta$-catenin reporter mouse (28) crossed to inducible endothelial cell-specific 
A

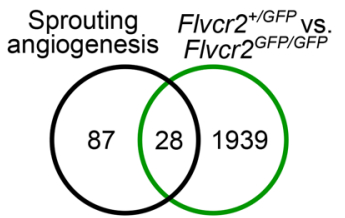

B
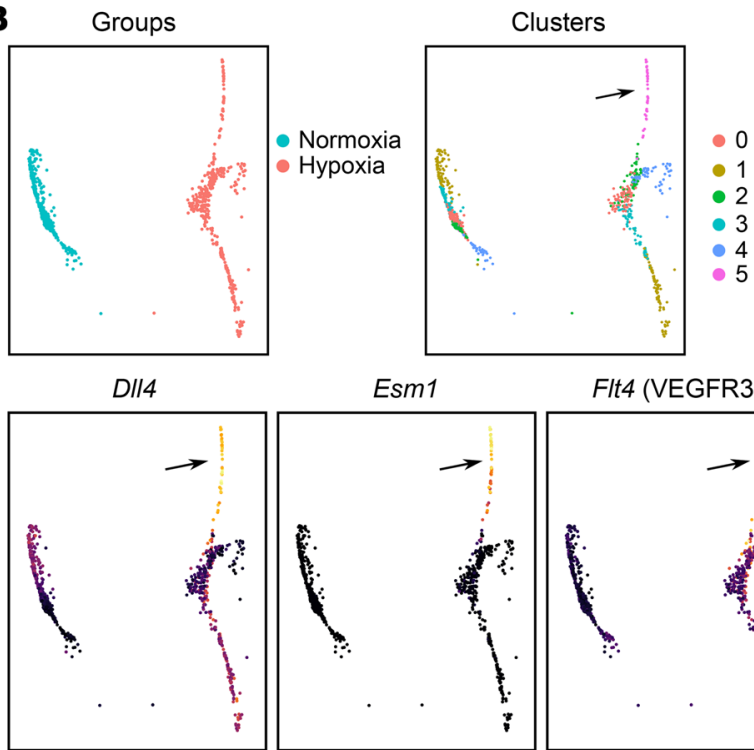

Emert

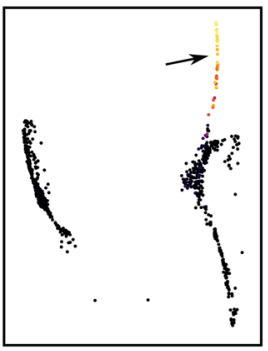

Low

D

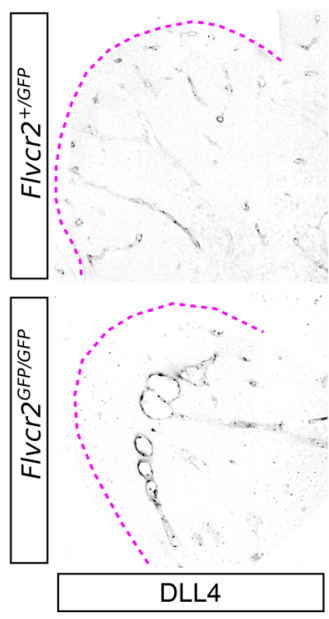

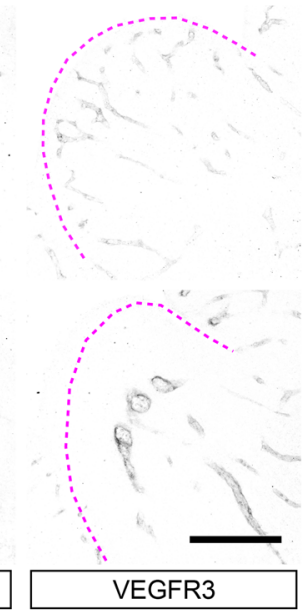
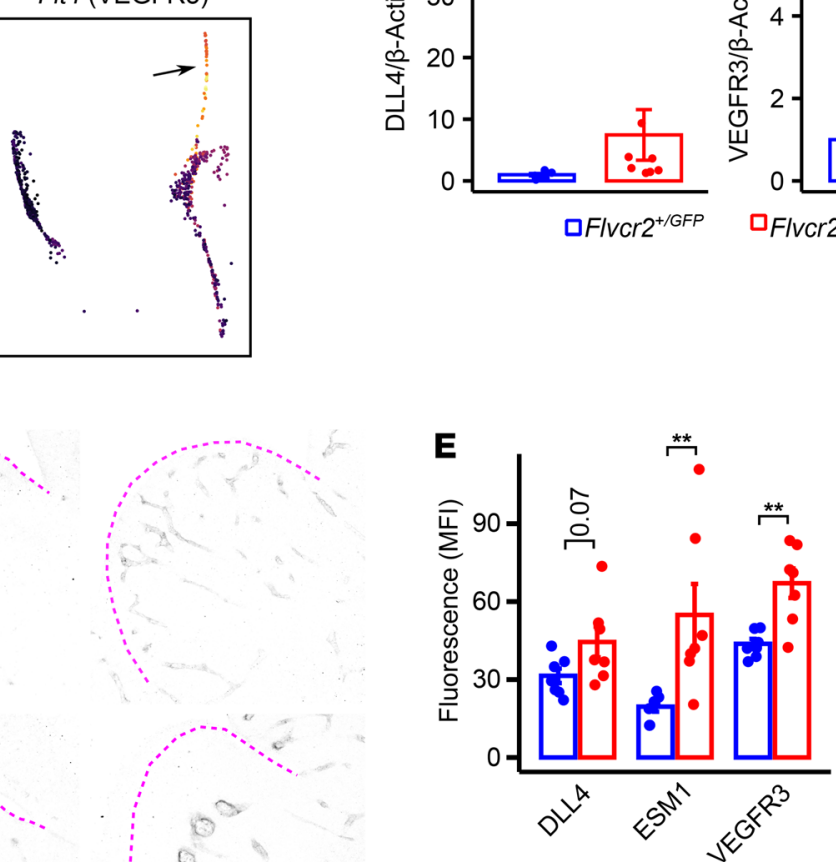

C
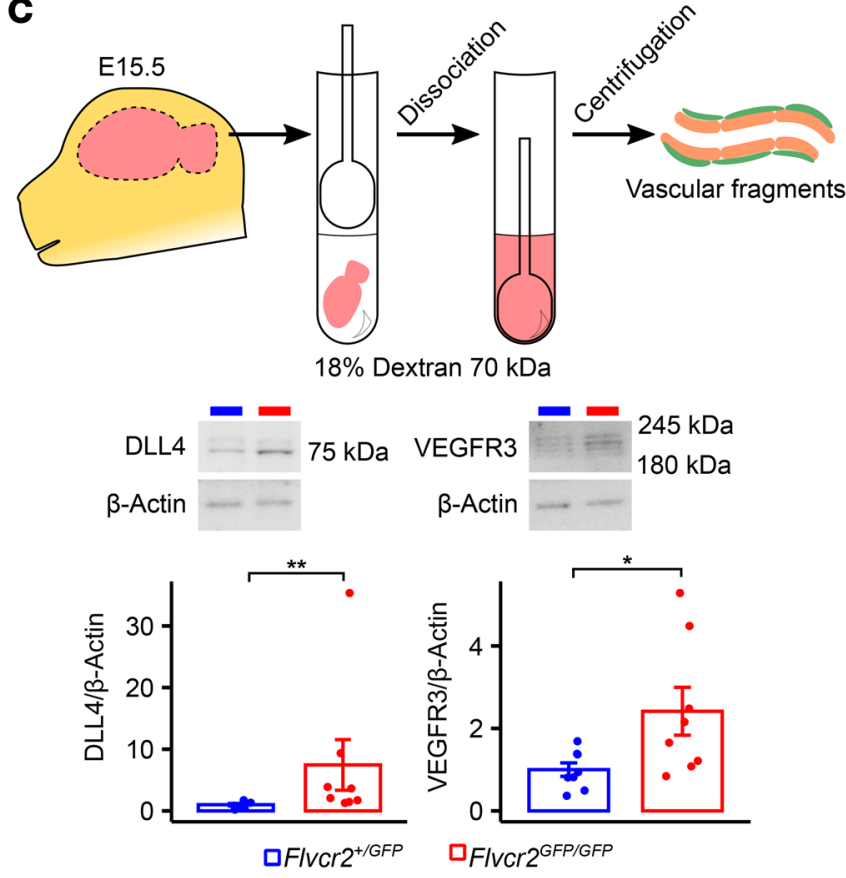

$180 \mathrm{kDa}$

B-Actin

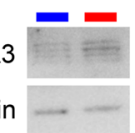

$245 \mathrm{kDa}$

口FIVCr2 ${ }^{\text {GFP/GFP }}$

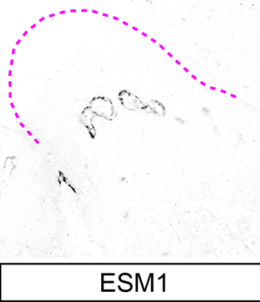

Figure 3. Angiogenesis is impaired by Flvcr2 inactivation. (A) Genes involved in sprouting angiogenesis that were differentially expressed in endothelial cells lacking Flvcr2 are shown. $n=4$ per genotype. (B) scRNA-Seq data from ref. 27 were reanalyzed (see Methods) to show expression levels of DII4, Flt4, and $E s m 1$ in endothelial cells from hypoxic brains. These genes were most highly expressed in a cluster unique to the hypoxic brain (cluster 5 , indicated by arrows). (C) Protein levels of angiogenic proteins were evaluated by Western blot in brain vascular fragments from E15.5 embryos. ${ }^{*} P<0.05 ;{ }^{* *} P<0.01, t$ test. $n=8$ per genotype. (D) Selected proteins were detected by immunofluorescence (shown in black over white background) in sections through the LGE at E14.5. Scale bar: $200 \mu \mathrm{m}$. (E) Fluorescence intensity of the selected proteins in isolectin-B4+ cells was quantified. ${ }^{*} P<0.01, t$ test. $n=7$ per genotype.

Flvcr 2 mutants or controls, we observed no evidence of repression (or activation) of WNT signaling (Supplemental Figure 6, C and D). Finally, we took a genome-wide approach and compared the transcriptional profiles of endothelial cells lacking Flvcr2, Tgfbr2, or Ctnnb1 (Supplemental Figure 6E). To inactivate Tgfbr2 in endothelial cells, we produced $T g f b r 2^{f / f l} ; R 26^{\text {TdTomato }}$; Cdh5-CreER embryos from dams injected with tamoxifen on days E11, E12, and E13. We sorted endothelial cells from these mice based on the expression of TdTomato and performed RNA-Seq in the same manner as with Flvcr2 $2^{\text {GFP/GFP }}$ endo- thelial cells. The transcriptional profile from Ctnnb1-deficient cells was obtained from the literature (3); in that study, authors performed targeted purification of polysomal mRNA-sequencing (TRAP-Seq) of brain endothelial cells at E14.5. By correlating these 3 data sets, we observed only limited similarity between Flvcr $2^{\text {GFP/GFP }}$ endothelial cells and the other 2 types of mutant endothelia (Supplemental Figure 6E).

Together, these data suggest that endothelial cells from Flvcr $2^{\text {GFP/GFP }}$ embryos maintain high levels of some elements of the response machinery to enforce angiogenic cues; however, the 


\section{Table 3. Change in expression levels of genes involved in sprouting angiogenesis in Flvcr2 ${ }^{\text {CFP/CFP }}$ versus Flvcr2 ${ }^{+/ / F P}$ embryos at E15.5}

\begin{tabular}{lc} 
Gene & Fold change \\
\hline Adamts9 & 4.82 \\
Anxa1 & 3.15 \\
Cdh13 & 2.36 \\
Dll4 & 1.43 \\
Eng & 1.50 \\
Epha2 & 2.66 \\
Esm1 & 15.18 \\
Fgfbp1 & 0.29 \\
Flt1 & 1.85 \\
Flt4 & 1.43 \\
Hmox1 & 0.62 \\
Itga5 & 1.45 \\
Itgb1 & 1.88 \\
Jak1 & 1.57 \\
Imjd6 & 1.50 \\
KIf2 & 0.38 \\
KIf4 & 0.38 \\
Loxl2 & 3.18 \\
Mmrn2 & 2.61 \\
Nr4a1 & 3 \\
Nrp1 & 1.74 \\
Pgf & 3.12 \\
Plk2 & 1.51 \\
Rhoj & 1.89 \\
Sema6a & 0.67 \\
Srpx2 & 5.33 \\
Tbxa2r & 1.57 \\
Vegfb & 0.45 \\
\hline
\end{tabular}

reduction in vessel ingression into the GE in the presence of high levels of VEGF suggests the mutant endothelial cells are unable to transduce those signals, independently of alterations in TGF- $\beta$ or $\mathrm{WNT} / \beta$-catenin signaling pathways.

Normally, tissue-derived VEGF gradients in growing or hypoxic tissue drive sprouting angiogenesis primarily through 2 distinct but related cellular processes: tip/stalk cell selection and endothelial proliferation (30). Endothelial cells at the angiogenic front are exposed to high levels of VEGF that signal through VEGFR2, enforcing an endothelial tip cell phenotype with numerous and elaborate filopodia. Further from the angiogenic front, endothelial cells are exposed to lower levels of VEGF and adopt stalk positions (31). During angiogenic growth, individual endothelial cells actively swap into tip and stalk cell positions (30) and simultaneously proliferate to generate more cells supporting the growing vascular structures. We were surprised to find that brain blood vessels in Flvcr 2 mutants failed to grow even in the presence of supraphysiologic VEGF levels and therefore sought to determine what aspect of sprouting angiogenesis might be affected in these mutants.

First, to identify proliferating endothelial cells in the embryo brain, we used KI67 and ERG immunostaining, which mark nuclei from cycling and endothelial cells, respectively (Figure 4A). We detected a reduction in $\mathrm{KI}_{67}{ }^{+}$endothelial cells at E14.5 in the GE of
Flvcr $2^{\text {GFP/GFP }}$, and we observed no change in proliferating endothelial cells at E12.5 (Figure 4B). However, the number of endothelial cells per vessel length was unchanged at both embryonic stages (Figure 4C). Since endothelial cells in the GE are exposed to high levels of VEGF at E14.5, these results support an impaired proliferative response to angiogenic cues in endothelial cells lacking Flvcr2.

Next, we considered whether tip/stalk cell selection could be affected by loss of Flvcr2. Consistent with an overall impaired endothelial response to angiogenic signals, we observed a strong reduction in the number (Figure 4, D and E) and length (Figure $4 \mathrm{~F}$ ) of filopodia produced by mutant $F l v c r 2^{\text {GFP/GFP }}$ versus control Flvcr2 $2^{+/ G F P}$ endothelial cells at the vascular front, both at E12.5 (before other vascular abnormalities) and E14.5.

The observed reduction in filopodia could be due to abnormal specification of endothelial cells in the correct position and/ or the failure of endothelial cells to sort to appropriate tip and stalk positions within a vascular segment. To differentiate these possibilities, we generated Flver 2 endothelial mosaics with sparse tamoxifen-inducible recombination in the Flvcr2 locus using Cdh5-CreERT2 (Flvcr2 $\left.{ }^{4 i E C}\right)$. We crossed $\mathrm{Flvcr}^{+/ f f} ; \mathrm{Cre}^{+}$females with Flvcr $2^{f / f l}$ males to obtain $\mathrm{Flvcr}^{f / f l f} ; \mathrm{Cre}^{+}$(Flvcr2 $2^{\operatorname{LiEC} / \Delta i E C}$; mutants) and Flvcr $^{+/ f l} ; \mathrm{Cre}^{+}$(Flvcr2 $2^{+/ \Delta i E C}$; controls) embryos within the same dam. Pregnant mice were injected with 3 doses of tamoxifen $(2 \mathrm{mg}$ per dose per animal) on consecutive days (E10.5, E11.5, E12.5). Since both mutant and control embryos carry the targeted allele, we took advantage of endogenous GFP reporter expression to identify mutant endothelial recombinants among nonrecombined WT cells in the LGE at E14.5. An important caveat to this approach is the possibility that some cells in Flvcr $2^{\Delta i E C / \triangle I E C}$ embryos may have only one recombined allele (i.e., heterozygous for $F l v c r 2^{G F P}$ ). Thus, our analyses may underestimate any phenotype associated with Flver2 inactivation. We defined "tip-like" positions as those within the first $50 \mu \mathrm{m}$ from the ventricle (Figure $4 \mathrm{G}$ ), based on the localization of the PVP, the greater number of filipodia per vessel length in this area (7), and the expression of prototypical markers such as ESM1 (Figure 1D and Figure 3B). We defined "stalk-like" positions as those outside of the tip-like position (Figure $4 G$ ). In Flvcr2 ${ }^{+/ \triangle i E C}$ embryos, the distribution of recombined cells was not different between the tip-like and stalk-like positions, as expected from unhampered sorting of recombined cells (Figure 4, $\mathrm{H}$ and I). However, in Flvcr ${ }^{4 i E C / \triangle I E C}$ embryos, recombined cells localized mainly to the stalk-like position (Figure 4, H and I). Taken together, these experiments indicate that endothelial cells lacking Flvcr2 are intrinsically impaired in their angiogenic capacity, likely due to a reduced ability to adopt a tip cell phenotype and an apparent inability to proliferate in response to supraphysiologic levels of VEGF. In the mosaic recombination setting, WT cells appear to compensate for Flvcr 2 mutant endothelium, which tend not to reach the tip position, allowing for relatively normal vascular network formation, with only a few exceptions (as illustrated in Supplemental Figure 7).

VEGFR2, the most important VEGF receptor in endothelial cells, promotes tip cell selection and endothelial proliferation (31). Furthermore, brain endothelial cell-specific deletion of VEGFR2 and antibody-mediated blockade of VEGFR2 in mouse embryos cause a vascular phenotype similar to that we observed in Flvcr2 mutants $(32,33)$. Based on this, we looked to determine how $K d r /$ VEGFR2 may be regulated in Flvcr2 mutant mice. We found $K d r$ 

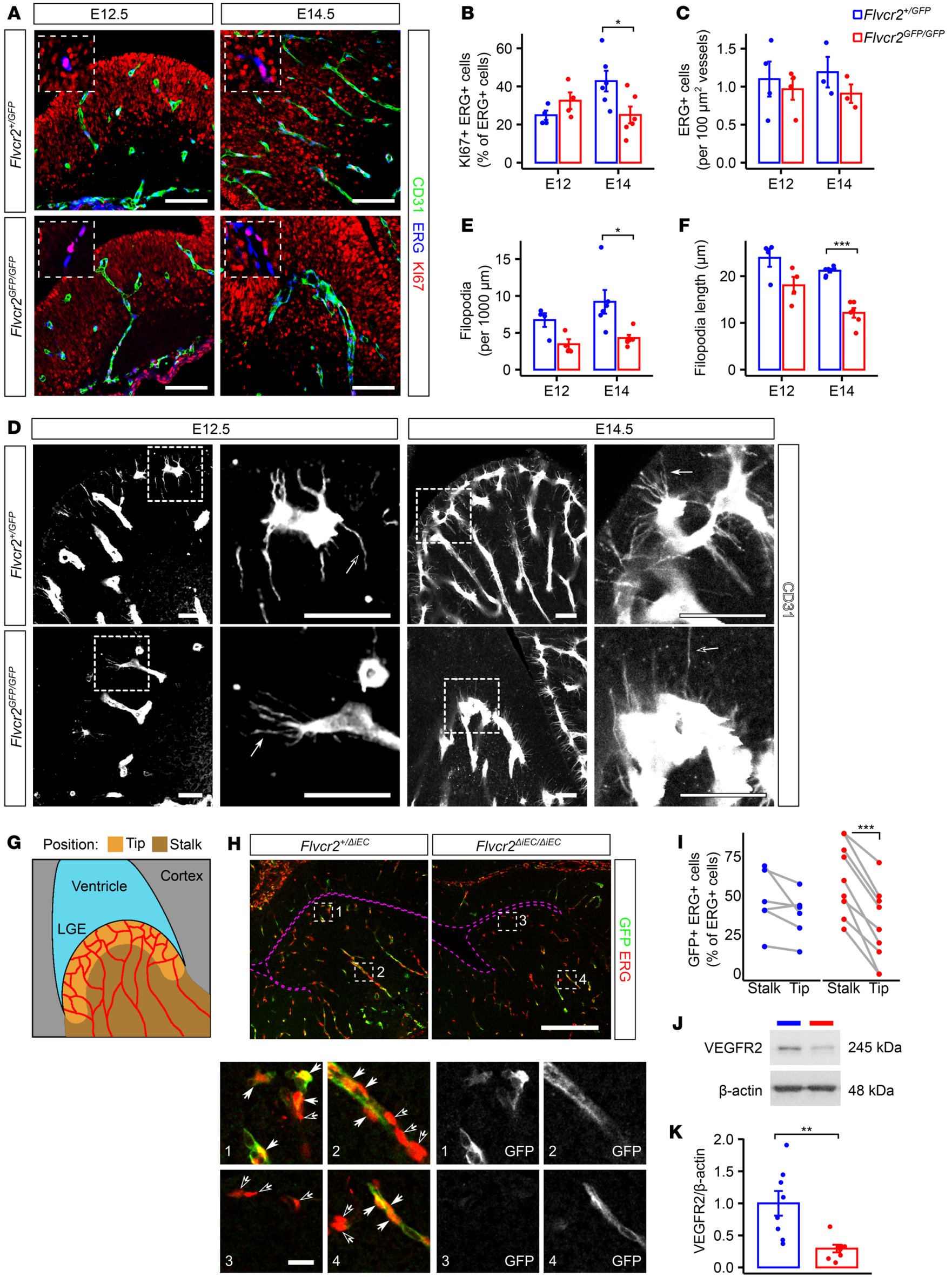
Figure 4. Flvcr2 regulates endothelial cell sprouting in the developing brain. (A) Coronal sections of the LGE at E12.5 and E14.5 were stained for CD31, the nuclear endothelial marker ERG, and the proliferation marker KI67. Scale bars: $100 \mu \mathrm{m}$. (B) Percentages of endothelial cells positive for the proliferation marker. ${ }^{*} P<0.05, t$ test. E12.5, $n=4$; E14.5, $n=6$. (C) Density of endothelial cells in the LGE. E12.5, $n=4$; E14.5, $n=3$. (D) Coronal sections containing LGE were stained with CD31 to reveal filopodia extending from endothelial cells (arrows). Scale bars: $50 \mu \mathrm{m}$. (E) Filopodia numbers were quantified as a function of $\mathrm{CD} 31^{+}$area at E12.5 and E14.5. ${ }^{*} P<0.05, t$ test. E12.5, $n=4$; E14.5, $n=6$. (F) Filopodia length was measured in the same sections. ${ }^{* *} P<0.001, t$ test. E12.5, $n=4$; E14.5, $n=6$. (C) Schematic representation of the vasculature in the LGE, highlighting presumed tip-like and stalk-like positions, based on the estimated localization of the PVP according to Figure $1 \mathrm{E}$. (H) Mosaic recombination was induced in Flvcr2 $2^{\mathrm{AiEC} /+}$ or Flver $2^{\text {AiEC/AiEC }}$ embryos by daily tamoxifen injections from E10 to E12. Inactivation of the Flver2 locus in endothelial cells was tracked by GFP production in $\mathrm{ERC}^{+} \mathrm{CD} 31^{+}$cells. Insets show magnifications of tip- and stalk-like positions. Open arrows mark GFP' cells, while closed arrows indicate GFP' cells. Scale bar: $250 \mu \mathrm{m} ; 20 \mu \mathrm{m}$ (inset). (I) Percentages of endothelial cells lacking Flvcr2 in the tip-like and stalk-like positions were quantified. ${ }^{* *} P$ $<0.001$, paired $t$ test. Flvcr2 ${ }^{\text {diEC/+}}, n=6$; Flvcr2 ${ }^{\text {diEC/AiEC }}, n=9$. (J) VEGFR2 was detected using Western blot in E15.5 brain vascular fragment homoge-

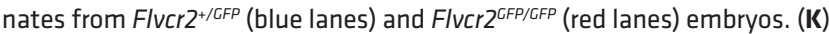
Quantification of VEGFR2 signal normalized to $\beta$-actin. ${ }^{* *} P<0.01, t$ test. $n$ $=8$ per genotype.

mRNA levels comparable to control in our RNA-Seq from isolated brain endothelial cells. However, there was a striking reduction in the protein levels of VEGFR2 in Western blots from isolated brain vascular fragments, suggesting that expression of VEGFR 2 is posttranscriptionally downregulated in mice lacking Flvcr2 (Figure 4, $\mathrm{J}$ and $\mathrm{K}$ ). Downregulation of VEGFR2 could be a primary consequence of reduced $F l v c r 2$ or secondary to its increased downregulation following hypoxia-driven expression of VEGF (34). Taken together, these results indicate that Flvcr2 expression is required for sprouting angiogenesis in the brain and that the vascular abnormalities observed in $F l v c r 2^{G F P / G F P}$ mutants may be a consequence of reduced VEGFR2-mediated endothelial cell sprouting.

Flvcr2 is dispensable for establishing and maintaining the $B B B$. Brain angiogenesis is very tightly coupled to the formation of the $\mathrm{BBB}$, and other mutant mice with vascular phenotypes similar to those of Flvcr ${ }^{\text {GFP/GFP }}$ embryos (e.g., WNT/ $\beta$-catenin pathway mutants, ref. 8; TGF- $\beta$ pathway mutants, ref. 7), also have defective BBB. Here, we tested BBB formation and maintenance in Flvcr2 mutant mice using markers of hemorrhage (extraluminal red blood cells) and various tracers of vascular leakage (Figure 5A). We used previously published $\mathrm{Tg} f b r 2^{f / f} ; \mathrm{Cdh} 5$ - $\mathrm{Cr} e^{E R T 2}$ embryos $\left(\mathrm{Tg} f b r 2^{\mathrm{AiEC} / \mathrm{AiEC}}\right.$ ), known to develop BBB defects and severe brain hemorrhage by E14.5 (7), as positive controls (Figure 5A). To our surprise, we found no evidence of hemorrhage or vascular leakage (10 kDa perfused dextran-TMR, endogenous IgG) in the brains of Flvcr2-deficient embryos at any analyzed stage, even in areas with severe vascular malformations or tissue infarction (Figure 5, A-C). Consistent with these results, immunostaining for canonical markers of BBB junctional protein CLDN5 (enriched in endothelia with an mature BBB) and PLVAP (enriched in fenestrated endothelia) in embryos revealed no change in these proteins in Flvcr $2^{G F P / G F P}$ mutants compared with controls (Supplemental Figure 8A). Furthermore, we observed no evidence of BBB dysfunction in adult Flvcr2 $2^{4 \mathrm{AEC} / \mathrm{ABEC}}$ mutants, using several different vascular tracers, including
sulfo-NHS-biotin, cadaverin-555, and Evans blue (Figure 5, D-H). Of note, $10 \mathrm{kDa}$ dextran-TMR and sulfo-NHS-biotin tracers were observed inside vascular lumens of perfused mice, confirming adequate distribution of the tracer as well as vascular lumenalization and perfusion of vessels in both $F$ lvcr2 ${ }^{\text {GFP/GFP }}$ embryos

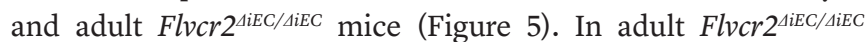
mice, there was no histological evidence of brain hemorrhage in any Flvcr 2 mutant and there were no major vascular abnormalities or other obvious phenotypic changes. It is worth noting that, with the tamoxifen regime used here, the possibility remains that some endothelial cells in Flvcr ${ }^{\text {diEC/AiEC }}$ still possess 1 intact allele, making them heterozygous. In order to attempt to address this issue, we took 2 distinct approaches. First, we induced gene inactivation at early postnatal days (P1, P2, and P3), a time at which other endothelial-specific tamoxifen-inducible mouse models have dysfunctional BBB (e.g., GPR124 mutants; ref. 28), and analyzed the $\mathrm{BBB}$ using the biotin tracer at P15. In this setting, we detected strong GFP signals in brain vessels, but we did not observe evident extravasation of the biotin tracer (Supplemental Figure 8B). Second, we crossed Flvcr $2^{f / f} ;$ C Cdh5-CreER mice with Flvcr2 $2^{+/ G F P}$ to obtain animals with 1 floxed allele and 1 null allele in the presence of the tamoxifen-dependent Cre recombinase $\left(F l v c r 2^{2 \mathrm{LiE} / G F P}\right)$. With this strategy, only 1 allele must be recombined to produce a mutant cell; however, the presence of the null allele in all cells precluded our ability to track recombination of the Flvcr2 locus, since all cells expressing Flvcr 2 would be $\mathrm{GFP}^{+}$. We induced recombination with 4 doses of tamoxifen and evaluated biotin leakage 20 days later. Again, we failed to observe leakage above control levels in the brain parenchyma (Supplemental Figure 8C).

Reduced pericyte coverage is associated with $\mathrm{BBB}$ defects (35), and a previous study documents reduced vascular mural cell staining in brain autopsy specimens from patients with Fowler syndrome (36). We observed normal pericyte coverage (area of CD31 vessels covered by NG2+ pericytes) at E12.5 and E14.5 and a slight reduction at E18.5 in Flvcr2 ${ }^{\text {GFP/GFP }}$ mutant embryos (Supplemental Figure 8D), suggesting that pericyte abnormalities are a mild, late, and likely a downstream event in PVHH pathogenesis and that these abnormalities are insufficient to affect BBB properties in Flvcr2 mutant mice. Taken together, these data indicate that Flvcr 2 is dispensable for the establishment and maintenance of the BBB in mice and is apparently dispensable for the maintenance of vascular morphology/structure in adult mice.

\section{Discussion}

Here, we report on the creation of a mouse model of PVHH that recapitulates the features of the human syndrome. We describe the associated vascular malformations and their consequences and provide evidence supporting a mechanism for their development involving impaired vascular sprouting due to reduced responsiveness to angiogenic cues. Interestingly, we observed a functional $\mathrm{BBB}$ in the mutants, using a comprehensive array of tracers.

Several mouse mutants have been described as presenting brain vascular malformations during embryonic development. These mutants disrupt distinct signaling pathways, including TGF- $\beta$ /integrin $\beta 8(7,37)$, neuropilin-1 (38), and WNT/ $\beta$-catenin $(8,28)$. Some of these pathways may interact through unknown mechanisms to drive brain angiogenesis, since their individual 


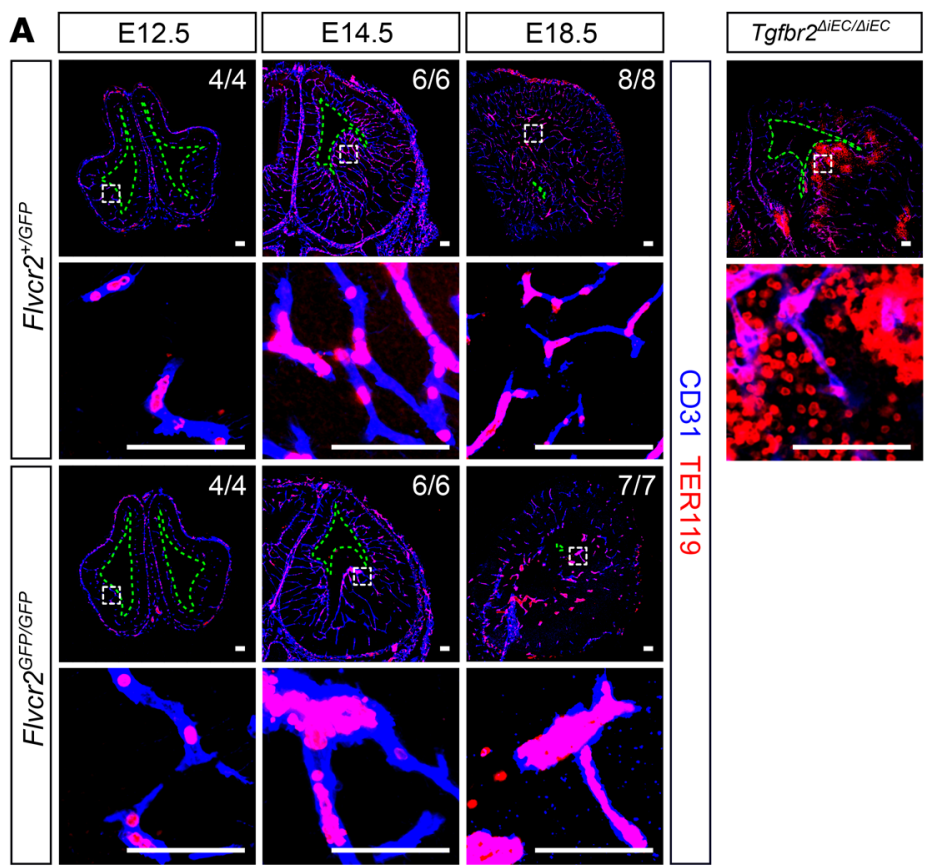

B
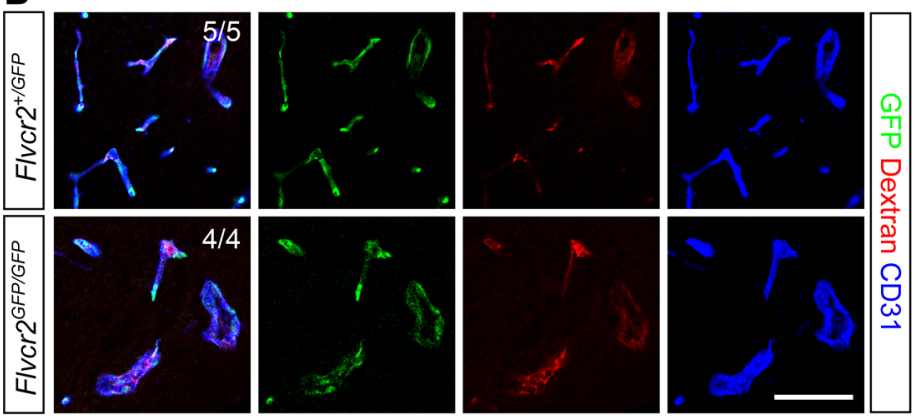

C
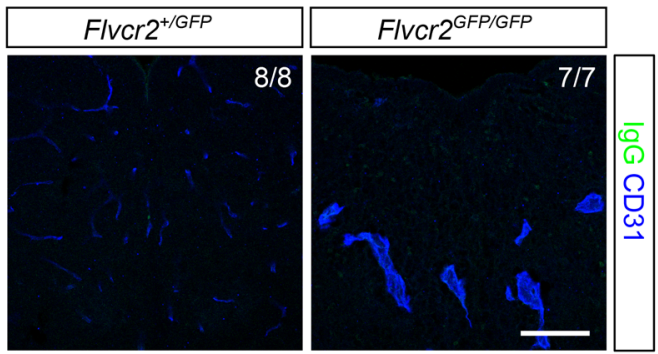

D

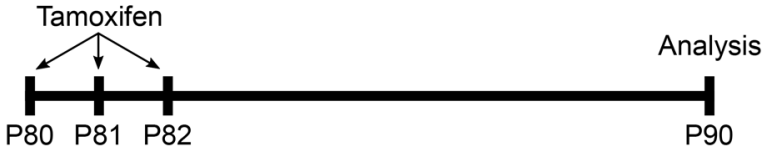

E

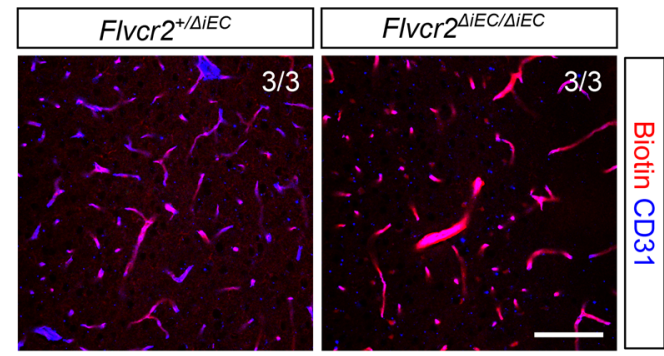

$\mathbf{F}$

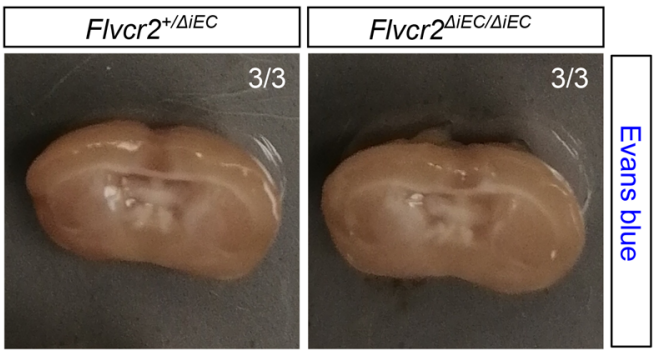

G

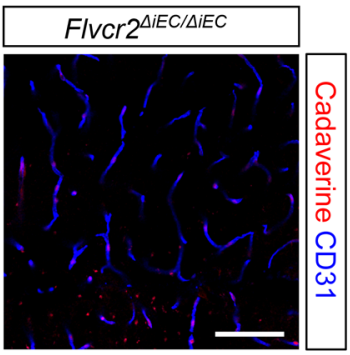

H

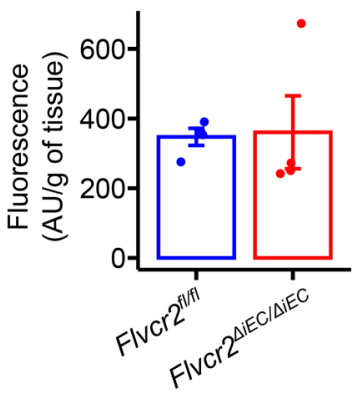

Figure 5. BBB is intact in Flvcr2 ${ }^{\text {CFP/CFP }}$ embryos. (A) Red blood cells were detected in embryonic brains using an antibody against TER119. Ventricles are delineated

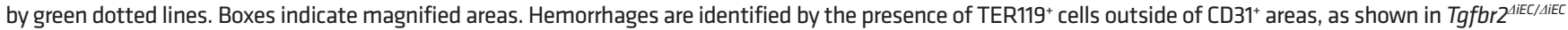
embryos. Representative image of 2 Tgfbr2 ${ }^{2 \text { iEC/AiEC }}$ embryos. Scale bars: $100 \mu \mathrm{m}$. (B) Embryos were injected intrahepatically with $10 \mathrm{kDa}$ dextran labeled with TMR at E18.5. The fluorescence of the labeled dextran was observed in brain sections stained for GFP and CD31 to test the ability of the tracer to leak into the brain parenchyma. Scale bar: $100 \mu \mathrm{m}$. (C) Endogenous mouse IgG was detected in brain sections at E18.5 with a fluorescently labeled antibody. Sections representative of brain parenchyma of the indicated number of embryos are shown. Scale bar: $100 \mu \mathrm{m}$. (D) Flvcr2 inactivation was induced by injecting tamoxifen in the indicated number of adult mice, and animals were perfused with sulfo-NHS-biotin 10 days after the last induction. (E) Biotin presence was detected with fluorescently labeled streptavidin in brain, and localization outside of CD31+ cells was assessed. Scale bar: $100 \mu \mathrm{m}$. (F) Recombination was induced in adult mice, and Evans blue was injected i.p. 10 days later. Brains were collected after perfusion and photographed intact. $n=3$. (G and $\mathbf{H})$ Fluorescently labeled cadaverine was injected i.v. into WT and Flvcr $2^{\text {AiEc/AiEC }}$ mice, and brains were collected 3 hours later. The brain from one animal was sectioned to visualize dye localization. Scale bar: $100 \mu \mathrm{m}$. Brains from cadaverine-injected mice were homogenized and tissue fluorescence was quantified. $n=4$.

disruptions lead to severe, yet very similar, phenotypes. Of special note, these mutants share widespread CNS vascular malformations, affecting all parts of the brain and the spinal cord even at early to mid stages (E14.5). Furthermore, they show massive hemorrhage in the brain parenchyma and/or disruption of the $\mathrm{BBB}$, depending on timing and degree of gene deletion (28). It is interesting that mouse mutants with isolated disruption of the $\mathrm{BBB}$ - without vascular malformations - do not present with extrava- 
sation of red blood cells (4-6). This has led to the conceptualization that vascular morphogenesis and stabilization are coupled; thus, blood vessel malformation and destabilization invariably lead to the spilling of luminal contents, including tracers and blood. Further support for this concept comes from pathological conditions such as cerebral cavernous malformation (CCM), which are associated with intracerebral bleeding in mice and humans (39). In the studies presented here, we challenge this view by demonstrating the presence of severe vascular malformations in the brain, without evidence of destabilization. We failed to find hemorrhage in the brains of Flvcr $2^{G F P / G F P}$ embryos, and we provide compelling evidence for a functional BBB in these mutants. Importantly, this indicates that downstream pathological consequences in Flvcr $2^{\text {GFP/GFP }}$ mice (hypoxia, tissue infarction, death) stem from impaired vascularization of the brain and not from secondary damage due to hemorrhage or vascular leak. We anticipate that this model, in combination with those mentioned above, will permit the study of brain angiogenesis separately from BBB maturation. This work may lead to potential therapeutic targets for the treatment of vascular disorders in the brain.

Humans with mutations in the FLVCR2 gene develop PVHH, with a phenotype similar to that observed in Flvcr $2^{\text {GFP/GFP }}$ embryos; however, disease presentation varies in timing, severity, and accompanying symptoms. Pathognomonic features of $\mathrm{PVHH}$ include hydrocephalus and vascular abnormalities ("glomeruloid vascular proliferation”) involving parenchymal blood vessels in various regions of the brain. Associated brain calcification and necrosis in the white matter, basal ganglia, brain stem, cerebellum, and spinal cord; other central nervous system malformations (e.g., hypoplastic cerebellum); retinal vascular abnormalities; and extraneural manifestations (ranging from intrauterine growth restriction to severe arthrogryposis with fetal akinesia sequence) are more variably present. While early descriptions and prenatal ultrasound provided evidence of hydranencephaly (absent cerebral hemispheres), postmortem examinations have more typically revealed a hemispheric mantle, indicating moderate to severe hydrocephalus. Based on histological examination of postmortem tissues, the vascular lesions are generally described as "glomeruloid" due to superficial resemblance to renal glomeruli. We believe that the term "proliferation" has been used descriptively; to our knowledge, there is no direct evidence of vascular cellular proliferation in PVHH lesions. With the exception of 2 reported cases of PVHH born alive, the disease causes mid- to late-gestation fetal demise. Compared with these descriptions of PVHH in humans, our mouse models with Flvcr 2 gene deletion consistently reproduce the most common features of human $\mathrm{PVHH}$, including severe brain vascular malformation, ventriculomegaly/hydrocephalus, and fetal or early postnatal demise. We did not find evidence of the more variable symptoms present in humans, such as fetal akinesia, and did not find evidence for vascular proliferation.

In human specimens, it has been impossible to determine the cause of the pathological findings, since there is no access to the time course of the disorder. However, some authors had speculated that the primary problem was that of the vasculature (36). Similarly to what occurs in humans with PVHH (12), we observed hydrocephalus/ventriculomegaly in Flvcr2 ${ }^{\text {GFP/GFP }}$ embryos, but without destruction of the cortex. We suspect that enlarged ven- tricles are a consequence of reduced tissue growth (hydrocephalus ex vacuo) stemming from abnormal vascularization, tissue hypoxia, and cell death. We show that endothelial cell-specific deletion of Flver 2 leads to a primary impairment in brain vascular sprouting. We propose that the disease is initiated in the more proliferative parts of the brain, which expand rapidly; therefore, vessels need to grow at a faster pace to provide appropriate oxygenation. A similar scenario has been proposed for the brain vascular malformations caused by neonatally induced endothelial-specific knockout of the small GTPase $C d c 42$; the resulting malformations affect mostly the cerebellum and the retina, i.e., parts of CNS that grow rapidly in the neonatal period (40).

The constitutive Flver2 inactivation studied here caused reduced brain vessel growth already in the embryo, leading to impaired perfusion of the rapidly growing germinal matrix, resulting in hypoxia and a subsequent massive increase in VEGF production. In fact, some or all of the gene expression changes present in Flvcr2 mutant endothelial cells at E14.5 may be a consequence of hypoxia. Comparing our transcriptomic data to single endothelial cell transciptomes from hypoxic brains revealed remarkable similarity. It is important to note that many of the genes common to both angiogenesis and hypoxia are in fact negative regulators of angiogenic sprouting and proliferation (e.g., DLL4 and high VEGF). Therefore, changes to these genes might not merely reflect the presence of brain hypoxia, but may actually drive the vascular response to it. For example, overexpression of VEGF in the developing mouse brain induces the formation of vascular abnormalities that are similar to those observed in Flvcr2 mutant mice (41). Paradoxically, brain endothelial-specific deletion of Vegfr2 or blocking of VEGFR2 with antibodies during early brain development also promotes the formation of similar ("glomeruloid") brain vascular abnormalities $(32,33)$, suggesting that VEGFR2 downregulation in the setting of high VEGF might lead to these vascular phenotypes. A similar phenomenon is observed in the retina, where high mitogenic stimulation by VEGF arrests the proliferation of angiogenic vessels (42). Therefore, it is possible that hypoxia-induced VEGF overproduction has the counterintuitive effect of potentiating the antiangiogenic effects of Flvcr2 disruption, exacerbating vascular malformation. VEGF overproduction might also be the cause of the reduction of VEGFR2 that we observe in Flver2 mutants, which is similar to what has been described in other studies (34).

If tissue hypoxia and resulting angiogenic abnormalities are part of Fowler syndrome pathogenesis, then what are the primary effects of Flvcr2 knockout that cause tissue hypoxia? To address this, we first focused on changes in blood vessels that occur before the onset of hypoxia. We found that periventricular endothelial cells had a marked reduction in filopodia number and length at E12.5, suggesting a primary problem with angiogenic sprouting in the brain and, in particular, the tip cells. This abnormality preceded all other observable changes. We then took advantage of the variable degrees of endothelial recombination in tamoxifen-inducible mutants to study this process in more detail. Importantly, in endothelial mosaics with low proportions of mutant/recombined endothelial cells, there was no evidence of hypoxia or glomeruloid vascular malformation. In these animals, we found that Flvcr2+/GFP (heterozygous) endothelial cells were distributed randomly throughout the brain vasculature, with equal contribu- 
tion to tip and stalk positions, whereas Flvcr2 ${ }^{\text {GFP/GFP }}$ (presumed to be homozygous knockout) mutant endothelial cells were found predominantly in the stalk position and less often in the tip position near the brain ventricle. We presume this is because WT endothelial cells compensate for mutant endothelial cells by preferentially taking the tip/ sprouting position, allowing for normal vascular growth. As would be predicted, in individual Flvcr2 mutants with a high burden of recombination (the most extreme example being the conventional null), there are inadequate numbers of WT endothelial cells to compensate for the presence of mutant endothelial cells, resulting in a more fulminant phenotype. Also, this scenario is analogous to what has been proposed for endothelial-specific Cdc 42 knockout, which leads to an inability of the tip cells to form filopodia and migrate to populate the peripheral parts of the developing retina. In mosaic situations in which Cdc42 endothelial cells are in the minority, those cells arrest and become overtaken by WT tip cells, resulting in the formation of an essentially normal vessel network. Only when $\mathrm{Cdc} 42^{-}$endothelial cells are in the majority and hence forced to occupy tip positions does angiogenic sprouting become impaired $(40,43)$.

Together, these results demonstrate an integral, cell-autonomous requirement for Flvcr2 in brain tip cell selection and angiogenic sprouting. Failure of angiogenic sprouting leads to subsequent hypoxia, with resultant activation of an angiogenic program (e.g., DLL4 and VEGFR3) that further exacerbates the vascular pathology when sprouting is deficient. Future studies using single cell transcriptomics in mosaic mutants should help to further distinguish primary from secondary vascular responses to Flvcr 2 mutation.

The molecular function of the FLVCR2 protein is controversial. A study suggests that it acts as a heme importer (11), in line with its homology and sequence similarity with FLVCR1, a heme transporter (44). Heme, the prosthetic moiety for numerous heme proteins, serves as a central regulator of oxygen transport (hemoglobin), aerobic metabolism (electron transport cytochromes), redox sensing, and gene transcription in erythroid and nonerythroid cells (45). Although the heme-transport function of FLVCR2 has not yet been tested in endothelium, we speculate that reduced endothelial cell heme due to mutation or knockout of Flvcr 2 might directly affect metabolic pathways involving heme and/or dysregulate transcriptional programs normally modulated by heme-binding transcription factors such as NR1D1/2 $(\operatorname{RevERB} \alpha / \beta)$ or BACH1 $(46,47)$. Of note, these transcription factors have been shown to regulate different aspects of angiogenesis $(48,49)$. An important next step will be to determine directly whether these heme pathways are affected by Flvcr 2 mutation and the effects of endothelial heme on vascular sprouting.

In conclusion, we have developed a mouse model of the rare disease PVHH that provides important insights into the regulation of brain angiogenesis by disentangling morphogenesis of this vascular bed from vessel stabilization.

\section{Methods}

Generation of the floxed Flvcr2 allele. Mice with a Flvcr2 FLEx (Flvcr2 $\left.{ }^{\text {GFP }}\right)$ allele were generated by Biocytogen. Briefly, the targeting vector was constructed as shown in Supplemental Figure 1A, linearized, and transfected into C57BL/6J embryonic stem (ES) cells (Beijing Biocytogen Co.) by electroporation. G418-resistant ES clones were screened for homologous recombination by PCR. Correctly targeted clones were confirmed by Southern blot analysis and sequencing. Two pos- itive clones were injected into BALB/c blastocysts and implanted into pseudopregnant females to generate chimeric mice. Chimeric mice were bred to $\mathrm{C} 57 \mathrm{BL} / 6 \mathrm{~J}$ mice to obtain F1 mice carrying the recombined allele containing the FLEx construct.

Animals. Animals carrying the Flvcr ${ }^{G F P}$ allele were maintained on a C57BL6 background in specific pathogen-free conditions at UCSF. All animals had access to food and water ad libitum. Flvcr2 ${ }^{+/ G F P}$ animals were generated by crossing to $\mathrm{Mef} 2 \mathrm{c}$-Cre mice (gift from Brian Black at Cardiovascular Research Institute, UCSF). Tek-Cre (Tg(Tek-cre)5326Sato) and Cdh5-CreER $R^{T 2}$ mice ( $\mathrm{Tg}(\mathrm{Cdh} 5$-cre/ERT2)1Rha; gift from Ralf Adams at Max Planck Institute for Molecular Biomedicine, Muenster, Germany) were used for endothelial cell recombination. Mice carrying the R26TCF/LEF-LSL-H2B-GFP allele (Gt(ROSA)26Sor ${ }^{\text {tm12(Tct/Lef-GFP*) Nat) }}$ were a gift from Jeremy Nathans at Johns Hopkins University (Baltimore, Maryland, USA). $T g f b r 2^{f / f l l}$ (Tgfbr2 ${ }^{\mathrm{tm} 1 \mathrm{Karl}}$ ) mice have been reported previously (50). The Ai14 allele (Gt(ROSA)26Sor ${ }^{\text {tm14(CAG-tdTomato)Hze } / J) ~ w a s ~ o b t a i n e d ~}$ from The Jackson Laboratory. DNA extracted from the mouse tails was analyzed by PCR for genotyping using primers in Supplemental Table 1 or as recommended by The Jackson Laboratory.

Embryo collection. For all crosses, one 2- to 6-month-old male was housed with two 2- to 4-month-old females and the presence of vaginal plugs was assessed every morning. Pregnant females were euthanized at the appropriate embryonic age (E12.5, E14.5, E15.5, or E18.5) by $\mathrm{CO}_{2}$ asphyxiation or isofluorane saturation and cervical dislocation. Some dams were injected i.p. with $180 \mathrm{mg}$ pimonidazole 3 hours before euthanasia. Uteri were retrieved from the abdominal cavity, and individual implantation sites were collected. Embryos were separated from the yolk sac and placenta, and the tail tip was saved for genotyping. Tissues were directly dropped in PFA $4 \%$ for fixation, flash-frozen, or further processed for vascular fragment separation.

Tamoxifen regimen. To induce cell-specific inactivation of the Flver 2 gene, adult mice were injected i.p. 3 times over 5 days with $2 \mathrm{mg}$ tamoxifen in corn oil. Alternatively, mice were injected 3 times on consecutive days on P60 and once more on P70 with $2 \mathrm{mg}$ tamoxifen. For juvenile inactivation, pups were injected intragastrically on P1, P2, and P3 with $0.2 \mathrm{mg}$ tamoxifen. To produce embryonic mosaic recombination, pregnant dams were injected with $2 \mathrm{mg}$ tamoxifen i.p. on days E10.5, E11.5, and E12.5.

Immunofluorescence. Embryonic tissues were fixed overnight in PFA $4 \%$, washed 3 times in PBS, and frozen in OCT to obtain $20 \mu \mathrm{m}$ sections in a cryostat. To obtain adult tissues for histological analyses, mice were perfused transcardially with PBS and then with PFA $4 \%$. Tissues were harvested and subsequently processed as embryonic tissue. Sections mounted onto glass were blocked and permeabilized for 2 hours in PBS containing 0.5\% Triton X-100, 1\% BSA, and 5\% normal donkey serum (blocking buffer) (MilliporeSigma). Next, sections were incubated overnight with primary antibodies diluted in blocking buffer in a humidified chamber. After 3 washes with $0.1 \%$ Tween-20 in PBS, sections were incubated with corresponding secondary antibodies in bocking buffer for 2 hours, washed again 3 times with 0.1\% Tween-20 in PBS, and mounted with CC/Mount medium (Diagnostics Biosyste$\mathrm{ms}$ ). Antibodies are described in Supplemental Table 2. Images were taken using a motorized Zeiss 780 upright laser-scanning confocal microscope with a 34-detector array with a water immersion Zeiss Plan Apochromat 20×/1.0, D = 0.17, parfocal length $75 \mathrm{~mm}$ (Zeiss). The spectral configuration was set up using the recommended settings from Zen software (Zeiss). Raw images were analyzed using ImageJ (NIH), and final figures were constructed with GIMP and Inkscape. 
Single-cell RNA-Seq reanalysis. Fastq files were first preprocessed using scPipe (51) to produce count matrix for each sample and to filter low-quality cells. Next, Seurat was used to regress out cell cycle effects, to integrate all samples with the SCTransformation, and to cluster cells with the Louvain algorithm (52). Cells were projected in a low-dimensional space using PHATE, and gene expression was imputed with MAGIC $(53,54)$. The complete $\mathrm{R}$ code for these steps is provided in Supplemental Methods.

Isolation of vascular fragments. Brains from E15.5 embryos were isolated in ice-cold PBS and disrupted lightly by 5 pestle strokes. Fragmented tissue was centrifuged at $1500 \mathrm{~g}$ for 20 minutes at $4^{\circ} \mathrm{C}$ and resuspended in 4 volumes of $18 \%$ dextran $70 \mathrm{kDa}$. Vascular fragments were pelleted by centrifugation at $1500 \mathrm{~g}$ for 20 minutes at $4^{\circ} \mathrm{C}$. Supernatant was further processed in this manner twice, and the 3 pellets were pooled together.

Western blot. Vascular fragments from embryos were lysed in RIPA buffer (25 mM Tris pH 7-8, $150 \mathrm{mM} \mathrm{NaCl}, 0.1 \%$ SDS, 0.5\% sodium deoxycholate, $1 \%$ NP-40) supplemented with protease inhibitor cocktail (Roche). Proteins were resolved in $10 \%$ or $8 \%$ acrylamide gels in denaturing conditions and transferred to PVDF membranes. Blots were blocked with 5\% BSA, 0.01\% Tween-20 in TBS for 2 hours, probed with primary antibodies (Supplemental Table 2) overnight at $4^{\circ} \mathrm{C}$ or for 1 hour at room temperature in blocking buffer, washed 3 times with $0.01 \%$ Tween-20 in TBS, incubated with corresponding secondary antibodies 1 hour at room temperature in blocking buffer, and washed 3 times as before. Bound antibody was revealed by chemiluminescence and imaged in a Chemidoc (Bio-Rad).

FACS. Embryos at E15.5 were retrieved as described above. Brains were dissected out in PBS, minced with scissors, and disgregated by mechanical dissociation followed by enzymatic treatment with DNAse $(1.5 \mathrm{mg} / \mathrm{mL})$ and collagenase/dispase $(3 \mathrm{mg} / \mathrm{mL})$ for 30 minutes at $37^{\circ} \mathrm{C}$. Dissociated cells were filtered through a $40 \mu \mathrm{m}$ cell strainer to exclude clumps and centrifuged at $300 \mathrm{~g}$ for 5 minutes. After one wash in PBS, the new pellet was resuspended in HBSS containing $5 \%$ FBS and stained with anti-CD31 conjugated to APC $(2 \mu \mathrm{m} / \mathrm{mL})$ and DAPI $(10 \mathrm{pg} / \mathrm{mL})$. Alternatively, cells from $\mathrm{Tg} f b r 2^{f / f l}$; Ai14; Cdh5-CreER embryos were sorted based on the endogenous TdTomato fluorescence. Cells were sorted on a FACSAria 3 cell sorter (BD Biosciences) directly onto RLT Plus buffer.

RNA-Seq. Total RNA was extracted from 2000 to 5000 sorted endothelial cells using the Rneasy Plus Micro Kit (QIAGEN). cDNAs were prepared from $20 \mathrm{ng}$ of total RNA using the SMART-Seq v4 Ultra Low Input RNA Kit (Takara Bio USA), and libraries were constructed from this cDNA with the Nextera XT DNA Library Prep Kit (Illumina). Quality of the libraries was checked by sequencing in a MiniSeq (Illumina), and equimolar amounts of each library were sequenced in a HiSeq 4000 (Illumina) using single-end $50 \mathrm{pb}$. Grooming of demultiplexed reads was performed with FASTQ groomer 1.04. Alignment to the mouse genome (mm10) was done using TopHat2 0.7. Transcript abundance estimation and differential expression significance testing were computed with Cufflinks 2.2.1. Heatmaps were constructed with Cluster 3.0 and visualized with TreeView 3.0beta01. Gene enrichment analysis of GO biological processes was performed with PANTHER 14.1.

$B B B$ testing. To test the functionality of the BBB in embryos, the liver from E18.5 embryos still attached to the placental circulation was exposed, and $5 \mu \mathrm{L}$ of $10 \mathrm{kDa}$ dextran at $4 \mathrm{mg} / \mathrm{mL}$ was slowly injected into the liver. Dextran was allowed to circulate for approximately 3 minutes, and the embryonic brain was isolated and fixed.
To assess the BBB in adult mice, animals were perfused transcardially with $10 \mathrm{mg}$ of EZ-Link sulfo-NHS-LC-biotin (Thermo Fisher) in PBS, followed by $4 \%$ PFA. Brains were collected and processed as described above for other tissues. Alternatively, mice were injected i.p. with $100 \mu \mathrm{L}$ of $2 \%$ Evans blue and euthanized 24 hours later for tissue collection. Finally, adult mice were injected i.v. with $0.17 \mathrm{mg}$ cadaverine-Alexa Fluor 555 (Thermo Fisher) in $100 \mu \mathrm{L}$ of PBS and euthanized 3 hours later. Mice were perfused transcardially with HBSS, and the forebrain and kidneys were lysed in 1\% Triton X-100 in PBS. Fluorescence intensity was determined using a fluorometer.

Data deposition. All sequencing data sets were deposited in the NCBI's Gene Expression Omnibus database (GEO GSE129838 and GSE146487). Previously published single cell RNA-Seq data from hypoxic brains can be found under accession GSE125708.

Statistics. The number of biological replicates was not defined using a statistical framework. To reduce bias, image analyses were performed using ImageJ macros where possible. Blinding to the genotype was not feasible at E14.5 and E18.5, since the phenotype is readily evident. Graphs show scatter dot plots with the mean \pm SEM. Deviations of genotype proportion from the expected value were tested using $\chi^{2}$ test. Statistical significance of differences between means was evaluated by $t$ test, which was performed because we only tested for the effect of genotype and not age. Paired $t$ test was used when comparing the difference in distribution of cells in mosaic embryos. A $P$ value of less than 0.05 was considered significant. All tests were 2-tailed and were performed using core functions in the R statistical environment, version 3.6.1.

Study approval. The studies presented here were conducted following the guidelines of the American Veterinary Medical Association (AVMA) and were approved by the Institutional Animal Care and Use Committee at UCSF.

\section{Author contributions}

NS, COL, EM, GLM, and BJ performed experiments and analyzed data. TDA, CB, and DS provided resources. TDA supervised the study. NS and TDA wrote the original draft. All authors contributed to and approved the final version of the manuscript.

\section{Acknowledgments}

We are indebted to Stephen Fancy for his help with AXIN2 Western blots. We thank the staff of the UCSF Genomics Core and the UCSF Center for Advanced Technology for their help in the RNASeq experiments. We thank the staff of the Laboratory for Cell Analysis at UCSF, which is supported by a National Cancer Institute Cancer Center Support Grant (P30CA082103). This work was funded by NIH/National Institute of Neurological Disorders and Stroke (KO8) (to TDA) and the Swedish Science Council, the Swedish Cancer Foundation, the Knut and Alice Wallenberg Foundation, and the Leducq Foundation (to $\mathrm{CB}$ ). NS is supported by American Heart Association (AHA) postdoctoral fellowship 20POST35120371.

Address correspondence to: Thomas D. Arnold, 1550 Fourth St., 548C, San Francisco, California 94158, USA. Phone: 415.476.5153; Email: thomas.arnold@ucsf.edu. Or to: Christer Betsholtz, Rudbecklaboratoriet, Uppsala, Sweden 751-85. Phone: 46.018.471.4816; Email: christer.betsholtz@igp.uu.se. 
1. Vasudevan A, Bhide PG. Angiogenesis in the embryonic CNS: a new twist on an old tale. Cell Adh Migr. 2008;2(3):167-169.

2. Sabbagh MF, et al. Transcriptional and epigenomic landscapes of CNS and non-CNS vascular endothelial cells. Elife. 2018;7:e36187.

3. Hupe M, et al. Gene expression profiles of brain endothelial cells during embryonic development at bulk and single-cell levels. Sci Signal. 2017;10(487):eaag2476.

4. Ben-Zvi A, et al. Mfsd2a is critical for the formation and function of the blood-brain barrier. Nature. 2014;509(7501):507-511.

5. Sohet F, et al. LSR/angulin-1 is a tricellular tight junction protein involved in blood-brain barrier formation. JCell Biol. 2015;208(6):703-711.

6. Nitta T, et al. Size-selective loosening of the blood-brain barrier in claudin-5-deficient mice. JCell Biol. 2003;161(3):653-660.

7. Arnold TD, et al. Excessive vascular sprouting underlies cerebral hemorrhage in mice lacking $\alpha \mathrm{V} \beta 8$-TGF $\beta$ signaling in the brain. Development. 2014;141(23):4489-4499.

8. Daneman R, Agalliu D, Zhou L, Kuhnert F, Kuo CJ, Barres BA. Wnt/beta-catenin signaling is required for CNS, but not non-CNS, angiogenesis. Proc Natl Acad Sci USA. 2009;106(2):641-646.

9. Meyer E, et al. Mutations in FLVCR2 are associated with proliferative vasculopathy and hydranencephaly-hydrocephaly syndrome (Fowler syndrome). Am J Hum Genet. 2010;86(3):471-478.

10. Khan AA, Quigley JG. Heme and FLVCR-related transporter families SLC48 and SLC49. Mol Aspects Med. 2013;34(2-3):669-682.

11. Duffy SP, et al. The Fowler syndrome-associated protein FLVCR2 is an importer of heme. Mol Cell Biol. 2010;30(22):5318-5324.

12. Fowler M, Dow R, White TA, Greer CH. Congenital hydrocephalus-hydrencephaly in five siblings, with autopsy studies: a new disease. Dev Med Child Neurol. 1972;14(2):173-188.

13. Radio FC, et al. Proliferative vasculopathy and hydranencephaly-hydrocephaly syndrome or Fowler syndrome: Report of a family and insight into the disease's mechanism. Mol Genet Genomic Med. 2018;6(3):446-451.

14. Thomas $\mathrm{S}$, et al. High-throughput sequencing of a 4.1 Mb linkage interval reveals FLVCR2 deletions and mutations in lethal cerebral vasculopathy. Hum Mutat. 2010;31(10):1134-1141.

15. Tabula Muris Consortium, et al. Single-cell transcriptomics of 20 mouse organs creates a Tabula Muris. Nature. 2018;562(7727):367-372.

16. Vanlandewijck M, et al. A molecular atlas of cell types and zonation in the brain vasculature. Nature. 2018;554(7693):475-480.

17. Fan X, Heijnen CJ, van der Kooij MA, Groenendaal $\mathrm{F}$, van Bel F. The role and regulation of hypoxiainducible factor-1alpha expression in brain development and neonatal hypoxic-ischemic brain injury. Brain Res Rev. 2009;62(1):99-108.

18. Nahrendorf M, Pittet MJ, Swirski FK. Monocytes: protagonists of infarct inflammation and repair after myocardial infarction. Circulation.
2010;121(22):2437-2445.

19. Hoyer FF, et al. Tissue-specific macrophage responses to remote injury impact the outcome of subsequent local immune challenge. Immunity. 2019;51(5):899-914.e7.

20. Fernández-López D, et al. Microglial cells prevent hemorrhage in neonatal focal arterial stroke. J Neurosci. 2016;36(10):2881-2893.

21. Fantin A, et al. Tissue macrophages act as cellular chaperones for vascular anastomosis downstream of VEGF-mediated endothelial tip cell induction. Blood. 2010;116(5):829-840.

22. Rosin JM, Vora SR, Kurrasch DM. Depletion of embryonic microglia using the CSF1R inhibitor PLX5622 has adverse sex-specific effects on mice, including accelerated weight gain, hyperactivity and anxiolytic-like behaviour. Brain Behav Immun. 2018;73:682-697.

23. Greenberg DA, Jin K. From angiogenesis to neuropathology. Nature. 2005;438(7070):954-959.

24. Lee HS, Han J, Bai HJ, Kim KW. Brain angiogenesis in developmental and pathological processes: regulation, molecular and cellular communication at the neurovascular interface. FEBS J. 2009;276(17):4622-4635.

25. Duran CL, et al. Molecular Regulation of Sprouting Angiogenesis. In: Pollock D, ed. Comprehensive Physiology. Hoboken, New Jersey, USA: John Wiley \& Sons; 2017:153-235.

26. Ribatti D, Crivellato E. "Sprouting angiogenesis", a reappraisal. Dev Biol. 2012;372(2):157-165.

27. Heng JS, et al. Hypoxia tolerance in the Norrin-deficient retina and the chronically hypoxic brain studied at single-cell resolution. Proc Natl Acad Sci USA . 2019;116(18):9103-9114.

28. Cho C, Smallwood PM, Nathans J. Reck and Gpr124 are essential receptor cofactors for Wnt7a/Wnt7b-specific signaling in mammalian CNS angiogenesis and blood-brain barrier regulation. Neuron. 2017;95(5):1221-1225.

29. Jho EH, Zhang T, Domon C, Joo CK, Freund JN, Costantini F. Wnt/beta-catenin/Tcf signaling induces the transcription of Axin2, a negative regulator of the signaling pathway. Mol Cell Biol. 2002;22(4):1172-1183

30. Chen W, et al. The endothelial tip-stalk cell selection and shuffling during angiogenesis. JCell Commun Signal. 2019;13(3):291-301.

31. Gerhardt $\mathrm{H}$, et al. VEGF guides angiogenic sprouting utilizing endothelial tip cell filopodia. JCell Biol. 2003;161(6):1163-1177.

32. Pu W, et al. Genetic targeting of organ-specific blood vessels. Circ Res. 2018;123(1):86-99.

33. Tan X, et al. Vascular influence on ventral telencephalic progenitors and neocortical interneuron production. Dev Cell. 2016;36(6):624-638.

34. Bruns AF, et al. Ligand-stimulated VEGFR2 signaling is regulated by co-ordinated trafficking and proteolysis. Traffic. 2010;11(1):161-174.

35. Armulik A, et al. Pericytes regulate the bloodbrain barrier. Nature. 2010;468(7323):557-561.

36. Harper C, Hockey A. Proliferative vasculopathy and an hydranencephalic-hydrocephalic syndrome: a neuropathological study of two siblings.
Dev Med Child Neurol. 1983;25(2):232-239.

37. Arnold TD, et al. Impaired $\alpha \mathrm{V} \beta 8$ and TGF $\beta$ signaling lead to microglial dysmaturation and neuromotor dysfunction. JExp Med.2019;216(4):900-915.

38. Gu C, et al. Neuropilin-1 conveys semaphorin and VEGF signaling during neural and cardiovascular development. Dev Cell. 2003;5(1):45-57.

39. Fischer A, Zalvide J, Faurobert E, Albiges-Rizo C, Tournier-Lasserve E. Cerebral cavernous malformations: from CCM genes to endothelial cell homeostasis. Trends Mol Med. 2013;19(5):302-308

40. Castro M, et al. CDC42 deletion elicits cerebral vascular malformations via increased MEKK3-dependent KLF4 expression. Circ Res. 2019;124(8):1240-1252.

41. Yang D, et al. Overexpression of vascular endothelial growth factor in the germinal matrix induces neurovascular proteases and intraventricular hemorrhage. Sci Transl Med. 2013;5(193):193ra90.

42. Pontes-Quero S, et al. High mitogenic stimulation arrests angiogenesis. Nat Commun. 2019;10(1):2016.

43. Laviña B, et al. Defective endothelial cell migration in the absence of Cdc42 leads to capillary-venous malformations. Development. 2018;145(13):dev161182.

44. Quigley JG, et al. Identification of a human heme exporter that is essential for erythropoiesis. Cell. 2004;118(6):757-766.

45. Sono M, Roach MP, Coulter ED, Dawson JH. Heme-containing oxygenases. Chem Rev. 1996;96(7):2841-2888.

46. Raghuram S, et al. Identification of heme as the ligand for the orphan nuclear receptors REV-ERBalpha and REV-ERBbeta. Nat Struct Mol Biol. 2007;14(12):1207-1213.

47. Ogawa K, et al. Heme mediates derepression of Maf recognition element through direct binding to transcription repressor Bach1. EMBOJ. 2001;20(11):2835-2843.

48. Jiang L, et al. Bach1 Represses Wnt/ $\beta$-catenin signaling and angiogenesis. Circ Res. 2015;117(4):364-375.

49. Li M, Gallo D, Csizmadia E, Otterbein LE, Wegiel B. Carbon monoxide induces chromatin remodelling to facilitate endothelial cell migration. Thromb Haemost. 2014;111(5):951-959.

50. Levéen $P$, et al. Induced disruption of the transforming growth factor beta type II receptor gene in mice causes a lethal inflammatory disorder that is transplantable. Blood. 2002;100(2):560-568.

51. Tian L, et al. scPipe: A flexible R/Bioconductor preprocessing pipeline for single-cell RNA-sequencing data. PLoS Comput Biol. 2018;14(8):e1006361.

52. Stuart T, et al. Comprehensive integration of single-cell data. Cell. 2019;177(7):1888-1902.e21.

53. Moon KR, et al. Visualizing structure and transitions in high-dimensional biological data. Nat Biotechnol. 2019;37(12):1482-1492.

54. van Dijk D, et al. Recovering gene interactions from single-cell data using data diffusion. Cell. 2018;174(3):716-729.e27. 